\title{
Constraining the Symmetry Parameters of the Nuclear Interaction
}

\author{
James M. Lattimer \\ Department of Physics and Astronomy, Stony Brook University, Stony Brook, NY 11794-3800, USA \\ and \\ Yeunhwan Lim \\ Department of Physics Education, Daegu University, Gyeongsan 712-714, Korea \\ james.lattimer@stonybrook.edu \\ yeunhwan.lim@gmail.com
}

\begin{abstract}
One of the major uncertainties in the dense matter equation of state has been the nuclear symmetry energy. The density dependence of the symmetry energy is important in nuclear astrophysics, as it controls the neutronization of matter in core-collapse supernovae, the radii of neutron stars and the thicknesses of their crusts, the rate of cooling of neutron stars, and the properties of nuclei involved in r-process nucleosynthesis. We show that fits of nuclear masses to experimental masses, combined with other experimental information from neutron skins, heavy ion collisions, giant dipole resonances and dipole polarizabilities, lead to stringent constraints on parameters that describe the symmetry energy near the nuclear saturation density. These constraints are remarkably consistent with inferences from theoretical calculations of pure neutron matter, and, furthermore, with astrophysical observations of neutron stars. The concordance of experimental, theoretical and observational analyses suggests that the symmetry parameters $S_{v}$ and $L$ are in the range $29.0-32.7 \mathrm{MeV}$ and $40.5-61.9 \mathrm{MeV}$, respectively, and that the neutron star radius, for a $1.4 \mathrm{M}_{\odot}$ star, is in the narrow window $10.7 \mathrm{~km}<R<13.1 \mathrm{~km}$ (90\% confidence). We can also set tight limits to the size of neutron star crusts and the fractional moment of inertia they contain, as well as the overall moment of inertia and quadrupole polarizability of $1.4 \mathrm{M}_{\odot}$ stars. Our results also have implications for the disk mass and ejected mass of compact mergers involving neutron stars.
\end{abstract}

Subject headings: dense matter — equation of state; dense matter — stars: stars - neutron stars

\section{Introduction}

The nuclear symmetry energy, and, in particular, its density dependence, has been the focus of much recent activity due to its importance to astrophysics (Lattimer and Prakash|2007). The expected neutrino signal 
from gravitational collapse supernovae within our Galaxy is sensitive to the symmetry energy both before core bounce (Swesty, Lattimer and Myra 1994) and at later times during the proto-neutron star stage (Roberts et al.2012). The symmetry energy near $n_{s}$, the baryon density at saturation, determines the radii (Lattimer and Prakash 2001) of neutron stars. Neutron star radii strongly influence the expected gravitational signals from mergers involving neutron stars (Bauswein and Janka 2012; Lackey et al. 2012). The symmetry en-

ergy also affects the crust's thickness and thermal relaxation time, observable in cooling and accreting (Page and Reddy 2012) neutron stars and in giant magnetar flares (Hurley et al. 2005; Thompson and Duncan 2001; Samuelsson and Andersson 2007). The composition of matter at densities above $n_{s}$, and the existence of neutrino processes which can rapidly cool neutron stars, depend on the symmetry energy (Lattimer et al. 1991), as do predicted properties of neutron-rich nuclei and reaction rates involved in the astrophysical r-process (Nikolov et al. 2011).

There have been many experimental attempts to constrain the nuclear symmetry energy, including measurements of neutron skin thicknesses, dipole polarizabilities, giant and pygmy dipole resonance energies, flows in heavy-ion collisions, and isobaric analog states (see, for example, recent summaries by Tsang et al. (2012); Newton et al. (2011)). These constraints are weakened by varying degrees of model dependence. Often overlooked, however, is the important relatively model-independent constraint arising from fitting nuclear masses, which comprise the most abundant and accurate data available. In combination with other experimental data, we show that including this mass constraint leads to tight bounds which are also compatible with astrophysical observations of neutron stars and theoretical studies of neutron matter.

\section{The Nuclear Symmetry Energy}

The nuclear symmetry energy, $S(n)$, is the difference between the bulk energy per baryon $(e(n, x))$ of pure neutron $(x=0)$ and isospin symmetric matter $(x=1 / 2)$ at baryon density $n$. We consider baryonic matter consisting of neutrons and protons only, and $x$ is the proton fraction. A related quantity is the densitydependent quadratic coefficient, $S_{2}(n)$, of an expansion of the bulk energy per baryon in the neutron excess $1-2 x$ :

$$
e(n, x)=e(n, 1 / 2)+S_{2}(n)(1-2 x)^{2}+\ldots
$$

A common approximation is to retain only the quadratic term in Equation 1, even for small proton fractions $x$, so that $S_{2}(n) \simeq S(n)$. For matter in nuclei, this approximation is valid, but care has to be taken at densities in excess of $n_{s}$, where the effects of higher-order-than-quadratic terms are amplified (Steiner 2006). Depending on the nuclear interaction, this can be important for comparisons with neutron star matter and with pure neutron matter.

It is customary to introduce the symmetry energy parameters

$$
\begin{aligned}
S_{v} & =S_{2}\left(n_{s}\right), \\
L & =3 n_{s}\left(d S_{2} / d n\right)_{n_{s}}, \\
K_{\text {sym }} & =9 n_{s}^{2}\left(d^{2} S_{2} / d n^{2}\right)_{n_{s}}, \\
Q_{s y m} & =27 n_{s}^{3}\left(d^{3} S_{2} / d n^{3}\right)_{n_{s}} .
\end{aligned}
$$


Although there are limited constraints on $K_{\text {sym }}$ from measurements of the giant isoscalar monopole resonance (Li et al. 2010), isotopic transport ratios in medium-energy heavy ion collisions (Chen et al. 2007), and neutron skin data from anti-protonic atoms (Centelles et al. 2009), the extraction of the symmetry contribution to the total incompressibility of nuclei and its separation into bulk $\left(K_{\text {sym }}\right)$ and surface contributions is still problematic. There are no experimental constraints on $Q_{\text {sym }}$. In contrast, there are several possible experimental constraints on values of $S_{v}$ and $L$.

\section{Nuclear Mass Fitting}

The most accurate and least ambiguous constraint comes from nuclear masses. Farine, Pearson and Rouben (1978) and Oyamatsu and lida (2003) showed that fitting Hartree-Fock and Thomas-Fermi nuclear models to mass data leads to a linear correlation between $S_{v}$ and $L$, but they did not explore its statistical significance. Lattimer (1992, 1996) and Danielewicz (2003) performed simplified statistical analyses utilizing the liquid droplet model. However, a more rigorous analysis was recently undertaken by the UNEDF collaboration (Bertsch, Dean and Nazarewicz 2007) using a sophisticated microscopic nuclear energy density functional (Kortelainen et al.2010). Before discussing their results, it is illustrative to infer the nature of this correlation through a two-step procedure involving the liquid drop model for nuclei and the properties of the nuclear surface. This illustration also demonstrates that the correlation between symmetry parameters determined from nuclear mass fits is not very model-dependent.

\subsection{Liquid Drop Model Illustration}

The standard liquid drop model (Myers and Swiatecki 1966) holds that the energy of a nucleus is approximately

$$
\begin{aligned}
E(Z, A) & =A\left(-B+S_{v} I^{2}\right)+A^{2 / 3}\left(E_{s}-S_{s} I^{2}\right)+ \\
& +\frac{3}{5} \frac{e^{2} Z^{2}}{r_{0} A^{1 / 3}}+E_{\text {shell }}(Z, A-Z)+E_{\text {pairing }}(A),
\end{aligned}
$$

where $B \simeq 16 \mathrm{MeV}$ is the bulk binding energy, per baryon, of symmetric matter, $E_{s} \simeq 19 \mathrm{MeV}$ is the symmetric matter surface energy parameter. $I=(A-2 Z) / A$ is the neutron excess, and $r_{0}=\left(4 \pi n_{0} / 3\right)^{-1 / 3}$ where $n_{0}$ is the nuclear saturation density. The last three terms in Eq. (3) represent the Coulomb, shell and pairing energies, respectively.

The net symmetry energy of an isolated nucleus is then

$$
E_{D, i}=I_{i}^{2}\left(S_{v} A_{i}-S_{s} A_{i}^{2 / 3}\right),
$$

so a linear correlation between $S_{v}$ and $S_{s}$ is expected from minimizing the differences between model predictions and experimentally measured symmetry energies, i.e., minimizing

$$
\chi^{2}=\sum_{i}\left(E_{\text {exp }, i}-E_{D, i}\right)^{2} /\left(\mathcal{N} \sigma_{D}^{2}\right),
$$


where $\mathcal{N}$ is the total number of nuclei and $\sigma_{D}$ is a nominal error. A $\chi^{2}$ contour one unit above the minimum value represents the $1-\sigma$ confidence interval which is an ellipse in this linear example.

The properties of the confidence ellipse are determined by the second derivatives of $\chi^{2}$ at the minimum,

$$
\begin{aligned}
{\left[\chi_{v v}, \chi_{v s}, \chi_{s s}\right] } & =\frac{2}{\mathcal{N} \sigma_{D}^{2}} \sum_{i} I_{i}^{4}\left[A_{i}^{2},-A_{i}^{5 / 3}, A_{i}^{4 / 3}\right] \\
& \simeq[61.6,-10.7,1.87],
\end{aligned}
$$

where $\chi_{v s}=\partial^{2} \chi^{2} / \partial S_{v} \partial S_{s}$, etc. The specific values follow from the set of 2336 nuclei with $N$ and $Z$ greater than 40 included in the Audi, Wapstra and Thibault (2003) table. The confidence ellipse in $S_{s}-S_{v}$ space has orientation $\alpha_{D}=(1 / 2) \tan ^{-1}\left|2 \chi_{v s} /\left(\chi_{v v}-\chi_{s s}\right)\right| \simeq 9.8^{\circ}$ with respect to the $S_{s}$ axis, with error widths

$\sigma_{v, D}=\sqrt{\left(\chi^{-1}\right)_{v v}} \simeq 2.3 \sigma_{D}$ and $\sigma_{s, D}=\sqrt{\left(\chi^{-1}\right)_{s s}} \simeq 13.2 \sigma_{D}$ where $\left(\chi^{-1}\right)$ is the matrix inverse. The correlation coefficient is $r_{D}=\chi_{v s} / \sqrt{\chi_{v v} \chi_{s s}} \simeq 0.997$. In this simple example, the shape and orientation of the confidence interval depend only on $A_{i}$ and $I_{i}$ and not the location of the $\chi^{2}$ minimum or other drop parameters.

Although the specific values of the best-fit parameters and the exact correlation are sensitive to the inclusion of terms representing shell, pairing, Coulomb diffusion, exchange and Wigner energies, deformations, and the neutron skin, among others, their effects are relatively minor. For example, Van Isacker and Dieperink (2006) showed that the Wigner term is important in identifying the best-fit values of the liquid drop parameters, but does not significantly affect the deduced correlation between $S_{v}$ and $S_{s}$.

\subsection{Liquid Droplet Model}

In practice, the liquid droplet model (Myers and Swiatecki 1969), which differs from the liquid drop model by accounting for varying neutron/proton ratios within the nucleus that produce neutron skins in neutron-rich nuclei, is an improved treatment. Its symmetry energy is

$$
E_{L D, i}=S_{v} I_{i}^{2} A_{i}\left(1+S_{s} A_{i}^{-1 / 3} / S_{v}\right)^{-1},
$$

and therefore predicts a linear correlation between $S_{s} / S_{v}$ and $S_{v}$ rather than between $S_{s}$ and $S_{v}$ as in the drop model. The same methodology as for the liquid drop model can be used to determine the confidence interval in $S_{s} / S_{v}-S_{v}$ space. In addition to $A_{i}$ and $I_{i}$, the properties of the confidence interval now depend, to a degree, on the measured masses, $E_{\text {exp }, i}$, as well as the parameters of the droplet model.

The correlation between $S_{s} / S_{v}$ and $S_{v}$ emerging from nuclear mass fitting is roughly equivalent to a correlation between $L$ and $S_{v}$ because the surface symmetry parameter, $S_{s}$, depends on the behavior of $S(n)$ within the nuclear interior. The general features can be appreciated using the plane-parallel approximation for the nuclear surface. At zero temperature, for densities less than the nuclear saturation density $n_{s}$, bulk matter separates into two phases of differing densities and proton fractions (a dense phase and a dilute phase) having equal pressures and neutron and proton chemical potentials. The surface thermodynamic potential per unit area, $\sigma$, also known as the surface tension, is the difference between the total thermodynamic potential and that of two uniform fluids each having the properties of matter far from the interface on both 
sides. It is straightforward to show that in the case appropriate for finite nuclei, in which the density in the dilute phase $(z=+\infty)$ vanishes,

$$
\sigma=2 \int_{-\infty}^{+\infty} n\left[e(n, x)-(1-x) \mu_{n}-x \mu_{p}\right] d z
$$

where $\mu_{n}$ and $\mu_{p}$ are the neutron and proton chemical potentials, respectively. To lowest order in the dense phase neutron excess $I=1-2 x_{-\infty}$, the surface tension can be expanded:

$$
\sigma \simeq \sigma_{0}-\left(1-2 x_{-\infty}\right)^{2} \sigma_{x}
$$

where $\sigma_{0}$ is the symmetric matter surface tension. In this notation, the surface energy parameters of the liquid drop and droplet models are $E_{s}=4 \pi r_{0}^{2} \sigma_{0}$ and $S_{s}=4 \pi r_{0}^{2} \sigma_{x}$.

One can show that to leading order (Lipparini and Stringari 1989; Danielewicz 2003; Steiner et al. 2005)

$$
\frac{S_{s}}{S_{v}}=\frac{E_{s}}{2} \frac{\int_{0}^{1} u^{1 / 2}\left[S_{v} / S_{2}(u)-1\right] f(u)^{-1 / 2} d u}{\int_{0}^{1} u^{1 / 2} f(u)^{1 / 2} d u},
$$

where $u=n / n_{s}$ and $f(u)=e(u, 1 / 2)+B$. A simple analytical relation is found if we approximate $S_{2}(u) \simeq$ $S_{v}+L(u-1) / 3$ and $f(u) \simeq K_{0}(u-1)^{2} / 18$, where $K_{0} \simeq 240 \mathrm{MeV}$ is the incompressibility parameter:

$$
\frac{S_{s}}{S_{v}} \simeq \frac{135 E_{s}}{2 K_{0}}\left[1-\eta \tan ^{-1} \eta^{-1}\right]
$$

where $\eta=\sqrt{3 S_{v} / L-1}$. Assuming $L / S_{v}=1,3 / 2$ or 2 , one finds, respectively, $S_{s} / S_{v} \simeq 0.7,1.2$ or 1.8 , which shows the basic trend although the values are somewhat less than given by realistic calculations.

This illustration is model-dependent, partly because it is based on the liquid droplet model and the semi-infinite surface approximation, but mostly because of the highly simplified assumed dependence of $S(n)$ on $L$ and $S_{v}$. In actuality, $S(n)$ depends on higher order terms such as $K_{s y m}$ and $Q_{s y m}$. We have found, applying the finite-range nuclear interaction (Myers and Swiatecki 1990) to Thomas-Fermi semi-infinite surface calculations, that $S_{s} / S_{v}$ can be approximated by

$$
\frac{S_{s}}{S_{v}} \simeq 0.6461+\frac{S_{v}}{97.85 \mathrm{MeV}}+0.4364 \frac{L}{S_{v}}+0.0873\left(\frac{L}{S_{v}}\right)^{2}
$$

with a mean error of $0.6 \%$. We found that a very similar relation emerges from Thomas-Fermi calculations using generalized Skyrme functionals. Very approximately, $S_{s} / S_{v}$ increases linearly with $L$, so that one expects the confidence interval in $L-S_{v}$ space to have a highly elongated elliptical shape with a small angle with respect to the $L$ axis.

Least-squares fitting of a liquid droplet model, together with the Thomas-Fermi result in Eq. 11, leads to best-fit values of the symmetry parameters $S_{v 0, L D}=29.1 \mathrm{MeV}$ and $L_{0, L D}=53.9 \mathrm{MeV}$. Shell effects were modeled with linear and quadratic terms in the neutron and proton valence numbers (the smallest distances from their respective magic numbers) as in Van Isacker and Dieperink (2006). Pairing effects were 
modeled with a term $a|A|^{1 / 2}$ for odd-odd nuclei, $-a|A|^{1 / 2}$ for even-even nuclei, and zero for odd-even nuclei, where $a \sim 12 \mathrm{MeV}$ was found from the fitting procedure. Conventional Wigner and Coulomb exchange energy terms were also employed, as was a further term representing the Coulomb diffuseness energy. The inclusion of these auxiliary terms, while improving the overall fit, which was $\chi_{\min } \sigma_{L D} \simeq 1.26 \mathrm{MeV}$, did not significantly alter the inferred correlation between $S_{v}$ and $L$, although the location of the $\chi^{2}$ minimum does depend on them. The results scale with the assumed value of the nominal error, in this case $\sigma_{L D}$. We also found that the properties of the confidence interval were $\sigma_{v, L D}=6.2 \sigma_{L D}, \sigma_{L, L D}=80.0 \sigma_{L D}$ and $\alpha_{L D}=15.3^{\circ}$ (Figure 11). In spite of the simplified nature of this model, its predictions turn out to be robust when compared to those of more sophisticated microscopic models.

\subsection{Mass Fit Correlations from Microscopic Nuclear Models}

The fitting of nuclear interaction parameters to binding energies using microscopic nuclear models was undertaken by Farine, Pearson and Rouben (1978). Using a number of different non-relativistic effective interactions, they performed a series of Hartree-Fock calculations and found a nearly linear correlation between $L$ and $S_{v}: L \simeq 10 S_{v}-290 \mathrm{MeV}$ (Figure 1). Later, Oyamatsu and Iida(2003) showed that fitting nonrelativistic and relativistic Hartree-Fock, as well as constrained variational Thomas-Fermi, nuclear models to mass data lead to the linear correlation $L \simeq 13.3 S_{v}-373.3 \mathrm{MeV}$ (Figure 1). Neither publication explored the statistical significance of their results. The slope of the Oyamatsu and lida (2003) correlation matches that of the liquid droplet model, but that of the Farine, Pearson and Rouben (1978) correlation is discrepant.

Kortelainen et al. (2010), simultaneously fitting nuclear masses and charge radii with the UNEDF0 potential and the Hartree-Fock methodology, explored the significance of the symmetry energy correlation. They obtained $S_{v 0, U N E D F 0} \simeq 30.5 \mathrm{MeV}, L_{0, U N E D F 0} \simeq 45.1 \mathrm{MeV}, \sigma_{v, U N E D F 0} \simeq 1.58 \sigma_{U N E D F 0} \mathrm{MeV}$, $\sigma_{L, U N E D F 0} \simeq 20.0 \sigma_{U N E D F 0} \mathrm{MeV}$ and $\alpha \simeq 15.2^{\circ}$. Kortelainen et al. (2010) adopted the somewhat arbitrary value of $\sigma_{U N E D F 0}=2 \mathrm{MeV}$, resulting in the $68 \%$ and $95 \%$ confidence intervals shown in Figure 1 . The RMS error of the UNEDF0 fit was $\chi \sigma_{U N E D F 0}=1.45 \mathrm{MeV}$.

This result closely matches fits obtained with both liquid droplet and Thomas-Fermi nuclear models (Figure 11. The length of the confidence interval for the liquid droplet model has the same size if we choose $\sigma_{L D}=0.50 \mathrm{MeV}$. For the finite-range Thomas-Fermi (FRTF) fits, the parameters for the shell, pairing, Wigner and Coulomb exchange energies were taken from the liquid droplet best-fit model rather than including them in the fitting procedure. We obtained $\chi \sigma_{F R T F} \simeq 2.75 \mathrm{MeV}, S_{v 0, F R T F} \simeq 31.2 \mathrm{MeV}$ and $L_{0, F R T F} \simeq 56.6 \mathrm{MeV}$. The length of the Thomas-Fermi finite-range confidence interval equals that of Kortelainen et al. (2010) if $\sigma_{F R T F}=3.1 \mathrm{MeV}$.

The correlation coefficient between $S_{v}$ and $L$ is approximately 0.97 for UNEDF0, less than the nearly unity value estimated from the liquid droplet or Thomas-Fermi models since the UNEDF0 model was also constrained by nuclear radii. It should be noted that the $95 \%$ confidence interval contains symmetry parameter values that may be somewhat unphysical from the point of view of the properties of neutron-rich matter

near and above the saturation density. For example, negative values of $L$ imply that pure neutron matter 
has negative pressures near $n_{s}$, implying stability for neutron clusters. The size of the confidence ellipse scales with the assumed value of $\sigma_{U N E D F 0}$, so had a value $\sigma_{U N E D F 0}=1 \mathrm{MeV}$ been chosen instead, the $95 \%$ confidence interval would have extended no lower than $L \simeq 20 \mathrm{MeV}$.

Another measure that can be applied to estimate the sizes of confidence intervals is the RMS error of the fit. For example, the liquid droplet $68 \%$ confidence interval displayed in Figure 1 corresponds to an RMS error of approximately $0.013 \mathrm{MeV} /$ baryon. In other words, applied to liquid drop dense matter equations of state, such as that of Lattimer and Swesty (1991), symmetry parameters chosen from within this confidence region would yield errors in neutron or proton chemical potentials of less than about $0.013 \mathrm{MeV}$ (aside from those arising from neglected shell and pairing effects).

The UNEDF collaboration has published a second parameter set, UNEDF1 (Kortelainen et al. 2012), for a modified energy density functional suitable for studies of strongly elongated nuclei. In this study, binding energies and charge radii, as well as excitation energies, of spherical nuclei, deformed nuclei and fission isomers, were fit. The best-fit values for the symmetry parameters of UNEDF1 lie near the $1-\sigma$ confidence ellipse of UNEDF0, as shown in Figure 1. However, this study did not discuss a correlation between $S_{v}$ and $L$. Because this fit is heavily weighted by deformed nuclei, and because the fit includes excitation energies, it will not show the same trends as the UNEDF0 fit.

As a final demonstration that this correlation is robust, the symmetry parameters determined from nuclear mass-fitting with the finite-range droplet model (FRDM) (Myers and Swiatecki 1990; Möller et al. 2012) has consistently produced values of $S_{v}$ and $L$ along the UNEDF0 correlation line (Figure 1). This occurs despite differences among nuclear models, including the algebraic forms of the nuclear interaction and treatments of shell, pairing, deformation and Wigner contributions. Unfortunately, confidence intervals for the FRDM fits were not published.

The correlation between symmetry parameters established from nuclear mass fitting has previously been used to constrain symmetry energy variations in supernova simulations (Swesty, Lattimer and Myra 1994), but, surprisingly, has rarely been combined with results derived from other experimental data. We show that doing so considerably enhances the constraints established from other experiments.

\section{Other Experimental Constraints}

The liquid droplet model implies that other nuclear observables will be related to the surface and volume symmetry coefficients and should provide additional constraints on them. Some of these constraints were summarized by Newton et al. (2011); Tsang et al. (2012).

\subsection{Neutron Skin Thickness}

An example is the neutron skin thickness of neutron-rich nuclei. Neglecting Coulomb effects, the difference between the mean neutron and proton surfaces in the liquid droplet model (Myers and Swiatecki 
1969) is

$$
t_{n p}=\frac{2 r_{o}}{3} \frac{S_{s} I}{S_{v}+S_{s} A^{-1 / 3}},
$$

which is, to lowest order, a function of $S_{s} / S_{v}$. Given that $S_{s} / S_{v}$ is largely a function of $L$, it should thus form a nearly orthogonal constraint to that from nuclear masses. Values of $t_{n p}$ have been measured, typically with $30-50 \%$ errors, for a few dozen nuclei. More frequently used is the neutron skin thickness $r_{n p}$, the difference between the mean square neutron and proton radii, which is approximately equal to $\sqrt{3 / 5} t_{n p}$.

A recent study of Sn isotopes (Chen et al. 2010), where differential isotopic measurements with fixed $Z$ potentially reduce errors, produced a correlation band in the $S_{v}-L$ plane which is nearly orthogonal to mass-fit correlations (Figure 22). The results from the $\mathrm{Sn}$ isotopes can, approximately, be expressed as a range of values of the neutron skin thickness of ${ }^{208} \mathrm{~Pb}, r_{n p}^{208}$, since numerous theoretical studies have shown them to be highly correlated (e.g., Reinhard and Nazarewicz (2010)).

A number of authors have demonstrated from Hartree-Fock and Thomas-Fermi studies that $r_{n p}^{208}$ varies linearly with $L$ for a given value of $S_{v}$ (see, for example, Furnstahl (2002)). However, $r_{n p}^{208}$ can also be shown to vary linearly with $S_{v}$ if $L$ is fixed (Furnstahl 2002; Reinhard and Nazarewicz 2010; Chen et al. 2010). In general, $r_{n p}^{208}$ is a function of both $S_{v}$ and $L$, and the high degree of correlation between $S_{v}$ and $L$ is responsible for these separate, nearly linear, correlations.

Chen et al. (2010) established a two-dimensional relation, $r_{n p}^{208}\left(S_{v}, L\right)$, using Skyrme Hartree-Fock calculations of ${ }^{208} \mathrm{~Pb}$ in which most Skyrme parameters were determined from nuclear matter properties, and $S_{v}$ and $L$ were then systematically varied. We can approximate their results with

$$
r_{n p}^{208}\left(S_{v}, L\right) \simeq a_{0}+a_{1} S_{v}+a_{2} L+a_{3} S_{v}^{2}+a_{4} S_{v} L+a_{5} L^{2},
$$

where the coefficients are given in Table 1. The mean error of this fit is about $0.7 \%$ over the ranges of interest. We attempted to reproduce the results for $r_{n p}^{208}\left(S_{v}, L\right)$ with a Skyrme Hartree-Fock analysis, and found similar results to within about $0.01 \mathrm{fm}$ over the relevant ranges of $S_{v}$ and $L$. The coefficients of our fit are also given in Table 1 . The fits are not identical due to differences in the assumed energy density functionals as well as errors in extracting values from Figure 7 of Chen et al. (2010). By combining the linear correlation between $L$ and $S_{v}$ from Kortelainen et al. (2010), namely $L \simeq-348.45 \mathrm{MeV}+12.903 S_{v}$, with Eq. 13 , we are able to reproduce the approximate relations between $r_{n p}^{208}$ and $L$ found by Furnstahl (2002); Chen, Ko and Li (2005); and Centelles et al. (2009).

Brown (2000) and Typel and Brown (2001) found an approximately linear correlation between $r_{n p}$ and the pressure $\left(p_{N}(0.1)\right)$ of pure neutron matter at the density $0.1 \mathrm{fm}^{-3}$,

$$
r_{n p}=(0.060 \pm 0.015)+0.12 p_{N}(0,1) .
$$

This correlation is phenomenological because matter in the neutron-rich nuclear surface is very different from pure neutron matter. $p_{N}(0.1)$ cannot be completely specified by $S_{v}$ and $L$, partly because of variations in the symmetric matter pressure and higher order terms in the expansion of $S_{2}$ with respect to density (see $\S 6.5$. Nevertheless, we have verified that Eq. [13, with the parameters in Table 1, reproduces this correlation. 
The limits on $r_{n p}^{208}$ deduced from the neutron skin thickness of $\mathrm{Sn}$ isotopes by Chen et al. (2010), $0.155 \mathrm{fm} \lesssim r_{n p}^{208} \lesssim 0.195 \mathrm{fm}$, yield the band shown in Figure 2. The above results indicate that this band is not very model-dependent.

A recent study (Krasznahorkay et al. 2013) of neutron skin thicknesses in $\mathrm{Sn}$ and $\mathrm{Pb}$ nuclei, deduced from measurements of antianalog giant dipole resonances, yielded the constraint $0.16 \mathrm{fm} \lesssim r_{n p}^{208} \lesssim 0.21 \mathrm{fm}$. Zenhiro et al. (2010), utilizing proton elastic scattering from ${ }^{208} \mathrm{~Pb}$, calculated $0.148 \mathrm{fm} \lesssim r_{n p}^{208} \lesssim 0.265 \mathrm{fm}$. Both agree with the analysis of Chen et al. (2010), but neither further constrains the symmetry parameters. Tsang et al. (2012) averaged results from various studies of photon emission during the decays of antiprotonic states of neutron-rich nuclei to deduce estimates for neutron skin thicknesses. They inferred a central value $r_{n p}^{208}=0.18 \mathrm{fm}$, but with even larger uncertainties. First results (Abrahamyan et al. 2012) from the PREX experiment to directly measure the neutron skin of ${ }^{208} \mathrm{~Pb}$ were too imprecise to be definitive, yielding $0.15 \mathrm{fm} \lesssim r_{n p}^{208} \lesssim 0.49 \mathrm{fm}$. The central value is discrepant with other results for neutron skins, but the uncertainties are still very large.

\subsection{Giant Dipole Resonance}

The hydrodynamical droplet model of Lipparini and Stringari (1989) showed that the giant dipole resonance (GDR) centroid energy is closely connected to liquid droplet parameters. In this model, the centroid energy is

$$
E_{-1} \simeq \sqrt{\frac{3 \hbar^{2}(1+\kappa)}{m<r^{2}>} S_{v}\left(1+\frac{5}{3} \frac{S_{s}}{S_{v}} A^{-1 / 3}\right)^{-1}},
$$

where $\kappa$ is an enhancement factor arising from the velocity dependence of the interaction and $<r^{2}>$ is the mean-square charge radius. The factor $m<r^{2}>/(1+\kappa)$ does not significantly vary among interactions for a given isotopic chain, and its value therefore does not play a role in estimating a correlation between the symmetry parameters. A series of measurements of the giant dipole resonance should result in a correlation between $S_{s} / S_{v}$ and $S_{v}$ that is similar to but slightly less steep (i.e., a greater value of $\alpha$ ) than that established

Table 1. Fitting coefficients for $r_{n p}^{208}$ in Eq. 13.

\begin{tabular}{lcc}
\hline \hline Coefficient & Chen et al. $(2010)$ & This paper \\
\hline$a_{0}$ & -0.094669 & -0.11767 \\
$a_{1}$ & 0.0072028 & 0.0070281 \\
$a_{2}$ & 0.0023107 & 0.0027521 \\
$a_{3}$ & $-8.8453 \cdot 10^{-6}$ & $4.7517 \cdot 10^{-7}$ \\
$a_{4}$ & $-4.7837 \cdot 10^{-5}$ & $-6.0221 \cdot 10^{-5}$ \\
$a_{5}$ & $4.0030 \cdot 10^{-6}$ & $4.9664 \cdot 10^{-6}$ \\
\hline
\end{tabular}


from nuclear masses. Equations (6) and (15) predict that the orientation angles $(\alpha)$ of the GDR and binding energy correlations in the $S_{s} / S_{v}-S_{v}$ plane will have the ratio

$$
\frac{\alpha_{G D R}}{\alpha_{\text {binding }}} \approx \frac{5}{3}\left(1+2 \frac{S_{s 0}}{S_{v 0}}<A^{-1 / 3}>\right)\left(1+\frac{8}{3} \frac{S_{s 0}}{S_{v 0}}<A^{-1 / 3}>\right)^{-1} \simeq 1.45,
$$

where $<>$ refers to an average over the measured nuclei. A similar ratio will then apply to the relative orientation angles in the $L-S_{v}$ plane.

Using a variety of Skyrme forces to model the giant dipole resonance in ${ }^{208} \mathrm{~Pb}$, Trippa, Coló and Vigezzi (2008) concluded the measured centroid energy was best fit by those forces having a bulk symmetry energy $S_{2}(0.1)$, evaluated at the density $n=0.1 \mathrm{fm}^{-3}$, in the range $23.3 \mathrm{MeV}<S_{2}(0.1)<24.9 \mathrm{MeV}$. The relation between $S_{2}(0.1)$ and $S_{v}$ and $L$ is obviously model dependent.

To explore this model dependence, we selected a number of functional forms for $S_{2}$ :

$$
\begin{aligned}
& S_{2}(u)=\left(T_{0} / 3\right) u^{2 / 3}(1+c u)+a_{1} u+b_{1} u^{4 / 3}, \\
& S_{2}(u)=S_{v}+(L / 3)(u-1)+\left(K_{s y m} / 18\right)(u-1)^{2}+\left(Q_{s y m} / 162\right)(u-1)^{3}+d_{1}(u-1)^{4}, \\
& S_{2}(u)=\left(T_{0} / 3\right) u^{2 / 3}+\left[S_{v}-\left(T_{0} / 3\right)\right] u^{\gamma},
\end{aligned}
$$

where $T_{0}=\left(\hbar^{2} / 2 m_{b}\right)\left(3 \pi^{2} n_{s} / 2\right)^{2 / 3}$ and

$$
\begin{array}{ll}
a_{1}=4 S_{v}-\frac{2 T_{0}}{3}\left(1-\frac{c}{2}\right)-L, & b_{1}=\frac{T_{0}}{3}(1-2 c)+L-3 S_{v}, \\
d_{1}=\frac{L}{3}-S_{v}-\frac{K_{s y m}}{18}+\frac{Q_{s y m}}{162}, & \gamma=\frac{3 L-2 T_{0}}{3\left(3 S_{v}-T_{0}\right)} .
\end{array}
$$

Form (i) of Eq. (17) originates from Skyrme interactions. Here, $c$ accounts for the nucleon effective mass in the kinetic energy contribution. We considered cases in which $c=0$ or $c=0.4$. Form (ii) is the polynomial density expansion with coefficients of Eq. (2). The parameter $d_{1}$ ensures that $S_{2}(0)=0$. To evaluate $K_{\text {sym }}$ and $Q_{s y m}$, which are not directly constrained by experiment, we considered two cases: Ducoin et al. (2011) argued them to be linearly correlated with $L$ for Skyrme functionals fit to nuclear properties:

$$
\begin{aligned}
K_{\text {sym }} & \simeq 3.33 L-281 \mathrm{MeV}, \\
Q_{\text {sym }} & \simeq-6.63 L+765 \mathrm{MeV},
\end{aligned}
$$

while Vidaña et al. (2009), using $Q_{\text {sym }}=0$, found

$$
K_{\text {sym }} \simeq 2.867 L-260 \mathrm{MeV} .
$$

This ensemble of four examples adequately describes the symmetry behavior of both Skyrme and relativistic field nuclear interactions. Finally, form (iii) has often been used in fitting results from heavy-ion collisions (Zhang et al. 2008).

The experimental constraints on $S_{2}(0.1)$ using these variations of Eq. (17) are displayed in Figure 3 , from which we infer that the effective model dependence is moderate. For example, the terms involving $Q_{s y m}$ and $d_{1}$ have little influence on these results. The form Eq. (17)iii) deviates at small values of $S_{v}$. We 
show in $\$ 6.5$ that this parameterization is a poor representation of neutron matter at subnuclear densities. To be conservative, however, we have chosen to adopt the extreme ranges to represent the experimental giant dipole resonance limits on $S_{v}$ and $L$ (Figure 2). The resulting band has a shallower slope, compared to that inferred from nuclear masses, as predicted.

\subsection{Dipole Polarizabilities}

The electric dipole polarizability, $\alpha_{D}$, is related to the response of a nucleus to an externally applied electric field, and it is largely concentrated in the giant dipole resonance. The approximate proportionality of $\alpha_{D}$ and $r_{n}^{208}$ was demonstrated macroscopically by Lipparini and Stringari (1989) and confirmed by microscopic calculations using energy-density functionals by Reinhard and Nazarewicz (2010). Tamii et al. (2011) measured the dipole polarizability of ${ }^{208} \mathrm{~Pb}$ to be $\alpha_{D}=(20.1 \pm 0.6) \mathrm{fm}^{3}$. Using the very high degree of correlation between $\alpha_{D}$ and $r_{n p}^{208}$ established by Reinhard and Nazarewicz (2010), this measurement of $\alpha_{D}$ is equivalent to the range $0.131 \mathrm{fm} \lesssim r_{n p}^{208} \lesssim 0.177 \mathrm{fm}$. The analysis by Reinhard and Nazarewicz (2010) was based upon a non-relativistic density functional analysis. A systematic reanalysis (Piekarewicz et al. 2012), incorporating both relativistic and non-relativistic models calibrated to nuclear properties in an attempt to minimize model dependencies, revised this permitted range to $0.146 \mathrm{fm} \lesssim r_{n p}^{208} \lesssim 0.190 \mathrm{fm}$. This result is nearly the same as that established from neutron skin thicknesses of $\mathrm{Sn}$ isotopes (Figure 2).

\subsection{Heavy Ion Collisions and Further Constraints}

Constraints from heavy ion collisions include those from isospin diffusion (Tsang et al.2009), shown in Figure 2, and from multifragmentation in intermediate-energy heavy ion collisions (Shetty, Yennello and Souliotis 2007). The latter implied $40 \mathrm{MeV}<L<125 \mathrm{MeV}$, which is consistent with our other constraints, but does not further limit parameter space and is therefore not displayed in Figure 2 for clarity. Both limits have been established from specific types of nuclear interaction models, and may be subject to considerable model dependence.

Also not included in Figure 2 are results of a study of pygmy dipole resonances (Carbone et al. 2010)

in ${ }^{68} \mathrm{Ni}$ and ${ }^{132} \mathrm{Sn}$, which implied $31<S_{v} / \mathrm{MeV}<33.6$ and $49.1<L / \mathrm{MeV}<80$. Daoutidis and Goriely (2011) argued that theoretical and experimental uncertainties prevent them from being effective constraints. The results of Reinhard and Nazarewicz (2010) confirmed that pygmy resonance energies lack a significant correlation with $L$.

Danielewicz and Lee (2009) proposed isobaric analog states as constraints on symmetry parameters and concluded that $S_{v} \simeq 33 \mathrm{MeV}$ and $S_{s} \simeq 96 \mathrm{MeV}$, which is equivalent to $L \approx 110 \mathrm{MeV}$. These values were derived from a fit including bulk and surface contributions to data for nuclei over a broad range of masses. However, we reanalyzed the fit including a curvature term (proportional to $A^{-2 / 3}$ ) and determined that $S_{v} \sim 30 \mathrm{MeV}$ and $S_{s} \sim 45 \mathrm{MeV}$, which would be equivalent to $L \approx 50 \mathrm{MeV}$. Neither fit is statistically preferred. A more detailed analysis will be necessary to produce better constraints. 
In summary, the various experimental results jointly confine the allowable ranges of symmetry parameters to a small region (Figure 2) bounded by $29.0 \mathrm{MeV}<S_{v}<32.7 \mathrm{MeV}$ and 40.5 MeV $<L<61.9 \mathrm{MeV}$. The lower limit to $L$ is set by the heavy-ion collision analysis, while the upper limit to $L$ is determined by the dipole polarizability analysis.

\section{Neutron Matter Theory}

Two recent studies of pure neutron matter have employed chiral Lagrangian (Hebeler et al. 2010) and quantum Monte Carlo (Gandolfi, Carlson and Reddy 2012) techniques using realistic two- and three-nucleon interactions. In each case, errors were estimated by considering physically-motivated ranges for interaction strengths. Symmetry parameters can be estimated from these calculations because, if higher-than-quadratic contributions in Eq1 1 can be neglected, the energy and pressure of pure neutron matter at the saturation density are $e\left(n_{s}, 0\right)=S_{v}-B$ and $p\left(n_{s}, 0\right)=L n_{s} / 3$, respectively. The horizontal width of the permitted quantum Monte Carlo region is underestimated because, in this calculation, $S_{v}$ was used as an input and was restricted to the indicated range $\left(31 \mathrm{MeV}<S_{v}<34 \mathrm{MeV}\right)$ based on nuclear mass fits. Despite their different theoretical approaches, these independent predictions for the symmetry parameters are remarkably consistent with each other and, furthermore, lie close to the experimentally preferred values (Figure 2).

Care should be taken with the neutron matter regions plotted in Figure 2 because they can be translated in both $S_{v}$ and $L$ if higher-than-quadratic terms exist in Eq. 1. In this case the quantity

$$
Q(n)=e(n, 0)-e(n, 1 / 2)-S_{2}(n)
$$

is non-zero and the value inferred for $S_{v}$ from the neutron matter energy at the saturation density, $e\left(n_{s}, 0\right)$, would be smaller by $Q\left(n_{s}\right)$ compared to the case in which higher-than-quadratic terms are ignored. The kinetic energy contribution to $Q$ for a non-interacting, non-relativistic, degenerate Fermi gas of nucleons, for example, is

$$
Q_{k}(n)=\frac{3 \hbar^{2}}{10 m_{b}}\left(2^{2 / 3}-\frac{14}{9}\right)\left(\frac{3 \pi^{2} n}{2}\right)^{2 / 3} \simeq 0.704\left(\frac{n}{n_{s}}\right)^{2 / 3} \mathrm{MeV} .
$$

The shift to lower inferred values for $L$ for this kinetic contribution would be $2 Q_{k}\left(n_{s}\right)$. Potential energy (and effective mass corrections) also contribute to $Q$. For many Skyrme and relativistic mean field interactions these are negligible by construction, but the MDI (momentum-dependent interaction) force (Das et al. 2003), often used in studies of heavy-ion collisions, has $Q\left(n_{s}\right) \simeq 0.27 S_{2}\left(n_{s}\right)$ and the implied shifts in $S_{v}$ and $L$ predicted by pure neutron matter are much larger than the kinetic energy contribution. If the shifts in $S_{v}$ and $L$ have the same sign, as they do for the kinetic energy contribution, the visible effect in Figure 2 would be small. However, the shifts could have opposite signs. More study of fourth-order corrections in the nuclear symmetry energy would be useful. 


\section{Discussion}

\subsection{Neutron Star Radii}

The most direct connection between astrophysical observations and the nuclear symmetry energy concerns neutron star radii $(R)$, which are highly correlated with neutron star pressures near $n_{s}$. Lattimer and Prakash (2001) found that the radii of neutron stars for masses near $1.4 \mathrm{M}_{\odot}$ obey a power law relation:

$$
R(M)=C(n, M)\left(p(n) / \mathrm{MeV} \mathrm{fm}^{-3}\right)^{1 / 4},
$$

where $R(M)$ is the radius of a star of mass $M, p(n)$ is the pressure of neutron star matter at density $n$, and $C(n, M)$ is a constant for a given density and mass. Lattimer and Prakash (2001) found that $C\left(n_{s}, 1.4 \mathrm{M}_{\odot}\right)=$ $9.30 \pm 0.60 \mathrm{~km}$ and $C\left(2 n_{s}, 1.4 \mathrm{M}_{\odot}\right)=5.72 \pm 0.25 \mathrm{~km}$. These values were estimated by averaging the results of 31 disparate equations of state, several of which are now ruled out because of the maximum mass constraint imposed by PSR J1614-2230 (Demorest et al.2010). Excluding those ruled-out equations of state, we find a revised value

$$
C\left(n_{s}, 1.4 \mathrm{M}_{\odot}\right)=9.52 \pm 0.49 \mathrm{~km},
$$

with a standard deviation $20 \%$ smaller than previously obtained. For comparison, at $2 n_{s}$, we find the fractional errors in the revised constant are now smaller by nearly a factor of 2: $C\left(2 n_{s}, 1.4 \mathrm{M}_{\odot}\right)=5.68 \pm 0.14 \mathrm{~km}$. Although this relation is a tighter fit when applied for densities somewhat in excess of the nuclear saturation density (e.g., $1.5-2 n_{s}$ ), neutron star matter pressure cannot be accurately predicted using the symmetry parameters $S_{v}$ and $L$ alone at these densities. Hence, we utilize Eq. (23), evaluated at $n_{s}$, for predictions.

The pressure of pure neutron matter at $n_{s}$ in the quadratic approximation (Eq. 1 is $p\left(n_{s}, x=0\right)=L n_{s} / 3$. However, neutron star matter is in $\beta$ equilibrium with a finite proton fraction and has a smaller pressure by a factor

$$
f \equiv \frac{p\left(n_{s}, x_{\beta s}\right)}{p\left(n_{s}, 0\right)}=1-x_{\beta s}\left[4\left(1-x_{\beta s}\right)-\frac{3 S_{v}}{L}\left(1-2 x_{\beta s}\right)\right] .
$$

$x_{\beta s}$, the proton fraction in $\beta$ equilibrium at the saturation density, is determined from $S_{v}$ by the cubic equation

$$
4 S_{v}\left(1-2 x_{\beta s}\right)=\hbar c\left(3 \pi^{2} n_{s} x_{\beta s}\right)^{1 / 3}
$$

For the range of values within the overlap region indicated in Fig. 2, $29.0 \mathrm{MeV}<S_{v}<32.7 \mathrm{MeV}$ and $40.5 \mathrm{MeV}<L<61.9 \mathrm{MeV}$, these relations imply $0.035<x_{\beta s}<0.046$ and $0.90<f<0.94$.

To estimate the radius and its error for $1.4 \mathrm{M}_{\odot}$ stars, we replaced the polygonal overlap region in Fig. 2 with an error ellipse such that Monte Carlo sampling would result in $68 \%$ of the points lying within the polygon. The ellipse has a semimajor (semiminor) axis of $6.7(0.55) \mathrm{MeV}$ and an orientation angle of $8.6^{\circ}$ from the vertical in the $S_{v}-L$ plane. With these specifications, we find that, with Eq. 24], $R_{1.4}=$ $11.9 \pm 0.7 \mathrm{~km}$ to $1 \sigma$ and $R_{1.4}=11.9 \pm 1.2 \mathrm{~km}$ to $90 \%$ confidence.

A study by Psonis, Moustakidis and Massen (2007), using the parameterized equation of state of Prakash, Ainsworth and Lattimer (1988) and assuming $S_{v}=30 \mathrm{MeV}$, found a nearly linear correlation between $R_{1.4}$ and $L, R_{1.4} \simeq 8.702+0.070 L \mathrm{~km}$, which agrees with Eqs. (23) and (24) to within their errors 
only for the interval $20 \mathrm{MeV}<L<60 \mathrm{MeV}$. The study of Gearheart et al. (2011) is only marginally consistent with predictions of Eqs. (23) and (24), perhaps because this study did not impose the neutron star maximum mass constraint.

Although more than 30 neutron star masses are now measured to a reasonable precision (Lattimer 2012), there are no precise measurements of neutron star radii. Among the most promising sources for determining radii are those stars having observed thermal radiation from their surfaces as well as certain X-ray bursts.

The interpretation of thermal emission has several difficulties, including uncertainties in distance, atmospheric composition, surface magnetic field, and interstellar absorption. Now-quiescent neutron stars in formerly accreting binaries in globular clusters (Heinke et al. 2006; Webb and Barret 2007; Guillot, Rutledge and Brown 2011) are promising because it is believed that recent accretion has both suppressed the surface magnetic fields and rendered their atmospheres with a nearly pure hydrogen composition. In addition, there have been significant advances in estimating distances to globular clusters. If these sources were true blackbodies, measured fluxes and temperatures would directly yield their apparent angular radii, $R_{\infty} / D$, where $R_{\infty}=R(1-2 \beta)^{-1 / 2}, \beta=G M / R c^{2}$ and $M$ is the stellar mass. These sources imply a wide range of values for $R_{\infty}$. A recent study found radii ranging from $8 \mathrm{~km}$ to $23 \mathrm{~km}$ (Guillot et al. 2013), using a low magnetic field, pure hydrogen atmosphere. Under the assumption that 5 quiescent sources would have a common radius, but possibly varying masses, motivated by the nearly vertical $M-R$ trajectories found by Steiner, Lattimer and Brown (2010, 2013) (see below), obtained a joint fit with $R=9.1_{-1.5}^{+1.3} \mathrm{~km}$ to $90 \%$ confidence, which is inconsistent with our results. However, this analysis has the theoretically unlikely result that, even with a common radius, the masses range from $0.86 \mathrm{M}_{\odot}$ to $2.42 \mathrm{M}_{\odot}$ (Guillot et al. 2013). Lattimer and Steiner (2013) suggest that uncertainties in the amount of interstellar absorption and atmosphere compositions contribute to the wide predicted ranges of mass and radius.

There are a few non-globular cluster sources having thermal emission, but only one, RXJ 1856-3954, has a precisely measured distance (Walter et al.2010). Ho et al. (2007) modeled it with a carbon-rich surface with a thin layer of hydrogen. Including only distance uncertainties, they found $M \simeq 1.33 \pm 0.09 \mathrm{M}_{\odot}$ and $R \simeq 11.9 \pm 0.8 \mathrm{~km}$. However, no confirmation of this assumed atmosphere composition is available.

Another class of sources, Type I photospheric radius-expansion (PRE) X-ray bursts (Strohmayer and Bildsten 2004), are thought to be powerful enough to temporarily lift material from neutron star surfaces. They have observed maximum fluxes thought to be near their Eddington limit values, $F_{E d d}=c G M /\left(\kappa D^{2}\right)$ (neglecting redshift), for which radiation pressure balances gravity ( $\kappa$ is the opacity). Combined with angular sizes determined from the tail of the burst, this permits simultaneous mass and radius determinations. For these sources, major uncertainties include distances as well as atmospheric properties. It is also uncertain if the location of the photosphere, when the maximum flux is observed, is close enough to the stellar radius to make redshift corrections to $F_{E d d}$ significant. Estimated errors in $M$ and $R$ are of order $20 \%$ or more for each source.

Steiner, Lattimer and Brown (2010) utilized $M-R$ data from observations of an ensemble of four quiescent and four PRE burst sources to estimate the parameters for an equation of state whose symmetry 
energy near the nuclear saturation density was parameterized by Eq. (17)iii). Their Bayesian analysis found, to $1 \sigma, 28.5 \mathrm{MeV} \lesssim S_{v} \lesssim 34 \mathrm{MeV}$ and $0.2 \lesssim \gamma \lesssim 0.4$, which implies $41 \mathrm{MeV} \lesssim L \lesssim 51 \mathrm{MeV}$. They also found, to $1 \sigma$ for their baseline case, $11.5 \mathrm{~km} \lesssim R_{1.4} \lesssim 12.2 \mathrm{~km}$. Alternatively, utilizing their derived constraints for $S_{v}$ and $L$, Eq. (23) predicts $11.0 \mathrm{~km} \lesssim R_{1.4} \lesssim 12.3 \mathrm{~km}$, which is quite consistent. In contrast, studies of PRE bursts by Özel and collaborators Özel, Baym and Güver (2010); Özel, Gould and Güver (2012), deduced radii with $2 \sigma$ uncertainties in the range 9-11 km. Also, Suleimanov et al. (2011) proposed that the short PRE bursts considered by Ozel et al. and Steiner et al. have systematic uncertainties, such as disc shadowing, that make them unsuitable for mass and radius determinations. They instead proposed the consideration of longer PRE bursts from which they find significantly larger radii, with estimates extending beyond $14 \mathrm{~km}$ ), which necessarily imply larger $L$ values (100 MeV or larger, according to Eq. (23), which are inconsistent with nuclear systematics. Collectively, these differences suggest that current models are subject to significant systematic uncertainties involving color correction factors, redshift corrections, and geometry.

A study by Steiner and Gandolfi (2012) combined neutron matter calculations and astrophysical observations to constrain $L$. They assumed the equation of state in the vicinity of the nuclear saturation density was parameterized by an energy density functional with parameters taken from the neutron matter results of Gandolfi, Carlson and Reddy (2012). They found, for neutron star models both with and without deconfined quark matter at high densities, that, to $1 \sigma, 43 \mathrm{MeV} \lesssim L \lesssim 52 \mathrm{MeV}$ and $32 \mathrm{MeV} \lesssim S_{v} \lesssim 34 \mathrm{MeV}$, and, to $2 \sigma, 39 \mathrm{MeV} \lesssim L \lesssim 54 \mathrm{MeV}$ and $31 \mathrm{MeV} \lesssim S_{v} \lesssim 34.5 \mathrm{MeV}$. To $1 \sigma$, they also inferred a neutron star radius range of $10.75-12.25 \mathrm{~km}$ for a $1.4 \mathrm{M}_{\odot}$ neutron star, only about $0.35 \mathrm{~km}$ smaller, on average, than obtained by Steiner, Lattimer and Brown (2010).

A more recent study (Steiner, Lattimer and Brown 2013) considered additional sources, and in addition considered systematic variations to the fiducial analysis in which one or more sources were excluded from the Bayesian analysis. In extreme cases, all PRE X-ray burst sources or quiescent globular cluster sources were excluded. With these variations in input data, it was inferred that $43.3 \mathrm{MeV} \lesssim L \lesssim 66.5 \mathrm{MeV}$ (Figure 2), to $1 \sigma$, somewhat larger on average than that deduced by Steiner, Lattimer and Brown (2010) and Steiner and Gandolfi (2012), but very close to the range found from experimental results. In agreement with the preceding studies, it was found that $S_{v}$ is relatively poorly constrained. The $1 \sigma$ radius range was found to be $10.6 \mathrm{~km} \lesssim R \lesssim 12.6 \mathrm{~km}$. The average value is the same as determined by Steiner, Lattimer and Brown (2010) but the range is broader due to the consideration of a greater variety of scenarios in an attempt to capture systematic uncertainties in models. It should be emphasized that neither Steiner, Lattimer and Brown (2010) nor Steiner, Lattimer and Brown (2013) assumed an apriori relation between the symmetry parameters, despite the fact that nuclear binding energies imply a strong correlation exists.

Finally, we note recent analyses (Leahy, Morsink and Chou 2011) of pulsed light from rotating, accreting millisecond pulsars are consistent with a mass-radius relation similar to that deduced by Steiner, Lattimer and Brown (2010). 


\subsection{Moments of Inertia and the Quadrupole Response}

The moment of inertia depends sensitively on the neutron star radius, scaling approximately as $R^{2}$. Hence, constraints to the neutron star radius will also constrain a neutron star's moment of inertia. The pulsar binary PSR 0737 (Lyne et al. 2004) has detectable spin-orbit coupling and the potential exists for a measurement of the most massive component's moment of inertia. Since the masses in this binary are known to a high degree of precision, this presents the possibility of measuring the radius of a neutron star. Lattimer and Schutz (2005) found that the moment of inertia, for neutron star equations of state capable of supporting at least $1.6 \mathrm{M}_{\odot}$, can be well approximated by

$$
I=(0.237 \pm 0.008) M R^{2}\left(1+2.84 \beta+18.9 \beta^{4}\right),
$$

where $\beta=G M / R c^{2}$ is the neutron star compactness parameter. For a $1.4 \mathrm{M}_{\odot}$ neutron star, this relation, coupled with the radius constraints implied by the experimental limits to $S_{v}$ and $L$, predicts $I_{1.4}=71.0 \pm$ $7.1 \mathrm{M}_{\odot} \mathrm{km}^{3}$. Another approximation for the moment of inertia (Link, Epstein and Lattimer 1999), after correction of a typographical error, is

$$
I \simeq \frac{2}{7} M R^{2}\left(1-\frac{33}{28} \beta\right)^{-1},
$$

which gives $I_{1.4}=71.4 \pm 7.2 \mathrm{M}_{\odot} \mathrm{km}^{3}$, a nearly identical result.

Flanagan and Hinderer (2008) have recently pointed out that tidal effects are potentially measurable during the late stages of inspiral of a binary merger involving neutron stars. Gravitational waves emitted early during the detection phase (when the orbital frequency becomes larger than about $100 \mathrm{~Hz}$ ) are nearly sinusoidal with gradually increasing frequency and amplitudes. Tidal forces induce quadrupole moments on the neutron stars, producing a very small correction which appears as an accumulated phase shift in the gravitational wave signal. This shift will be proportional to a weighted average of the induced quadrupole polarizabilites ( $\lambda_{\text {tid }}$, also known as the Love number) for the individual stars. Since both neutron stars have the same equation of state, this weighted average is relatively insensitive to the binary mass ratio (Hinderer et al. 2010). Since $\lambda$ scales as $R^{5}$, it would provide a sensitive measurement of the neutron star radius. However, the results of Hinderer et al. (2010) and Postnikov, Prakash and Lattimer (2010) show that for radii restricted to our favored range, $\lambda_{\text {tid }}$ is small, making detection of the tidal signature by Advanced LIGO difficult (Hinderer et al. 2010). Much of the difficulty has to do with degeneracies between the spins of the neutron stars, their rotational quadrupole moments $\left(Q_{r o t}\right)$ and their tidal Love numbers $\lambda_{\text {tid }}$ in their extraction from gravitational wave signals.

However, Yagi and Yunes (2013) pointed out that nearly EOS-independent relations exist among $Q_{r o t}$, $I$ and $\lambda_{\text {tid }}$ which can be used to break these degeneracies. In particular, the phenomenological relations Yagi and Yunes (2013) found relating $I$ to $\lambda_{\text {tid }}$ and $Q_{r o t}$ have interesting consequences. These are

$$
\begin{aligned}
\bar{I} \equiv I / M^{3} & \simeq \exp \left(1.47+0.817 z+1.49 z^{2}+0.287 z^{3}-0.364 z^{4}\right), \\
\bar{Q}_{r o t} \equiv Q_{r o t} / M^{5} & \simeq \exp \left(0.194+.936 z+.474 z^{2}-4.21 z^{3}+1.23 z^{4}\right)
\end{aligned}
$$


where $z=\left(\ln \bar{\lambda}_{\text {tid }}\right) / 10$ and $\bar{\lambda}_{\text {tid }}=\lambda_{\text {tid }} / M^{5}$. It is claimed these relations are accurate to better than a percent for all realistic EOSs and for neutron star masses greater than about $0.5 \mathrm{M}_{\odot}$, although they studied only four EOSs. We have verified the general validity of this relation, although we find it holds to slightly lesser accuracy than claimed. We find this relation holds to $2 \%$ accuracy for all the hadronic EOSs described in Lattimer and Prakash (2001) for stars greater than $1 \mathrm{M}_{\odot}$, except for PS, WFF3 and WFF4. For the latter EOSs, none of which satisfy the minimum maximum mass constraint, the error increases beyond $2 \%$, but only for $M<1.4 M_{\odot}$.

These equations imply that, for a $1.4 \mathrm{M}_{\odot}$ star, $\bar{\lambda}_{\text {tid }} \simeq 72 \pm 32$ and $\bar{Q}_{\text {rot }} \simeq 3.13 \pm 0.45$.

These relatively EOS-independent relations can be combined with Eq. 27), which itself is accurate to about $3 \%$ for the same mass range and the same set of EOSs, excluding those that cannot satisfy the minimum maximum mass constraint imposed by PSR 0737. This results in relations relating $\bar{\lambda}_{\text {tid }}$ and $\bar{Q}_{\text {rot }}$ to the compactness $\beta$ alone. We determined inverse relationships for Eq. 29] for this purpose:

$$
\begin{aligned}
& \bar{\lambda}_{\text {tid }} \simeq \exp \left(-4.24+5.96 u-3.01 u^{2}+0.722 u^{3}-0.0655 u^{4}\right), \\
& \bar{Q}_{r o t} \simeq \exp \left(-1.16-0.700 u+1.83 u^{2}-0.593 u^{3}+0.062 u^{4}\right),
\end{aligned}
$$

where

$$
u=\ln (\bar{I}) \simeq \ln \left[0.237 \beta^{-2}\left(1+2.84 \beta+18.9 \beta^{4}\right)\right] .
$$

We confirmed that these relations are valid to within about $3 \%$ for masses larger than $1 \mathrm{M}_{\odot}$ and $\beta \lesssim 0.3$ for EOSs that satisfiy the minimum maximum mass constraint. Maselli et al. (2013) points out that a measurement of $\lambda_{\text {tid }}$ to even $60 \%$ accuracy can be used to determine $\beta$ to $10 \%$ percent accuracy, because $\lambda_{\text {tid }} \propto R^{5}$. This would translate into a similar accuracy for the radius assuming the masses can be determined to at least this accuracy. However, we note that a simpler expression for $\bar{\lambda}_{\text {tid }}(\beta)$ obtained by Maselli et al. (2013) is considerably less accurate than Eq. (30) when applied to the same suite of EOSs and for the same conditions on mass and compactness.

\subsection{Binding Energies}

One of the more accurate measurements that can be determined from the neutrino signal from a galactic gravitational collapse supernova is the neutron star binding energy, assuming the distance to the supernova can also be found. Lattimer and Prakash (2001) determined a fit to the binding energy (BE) of a neutron star, for EOSs which support at least $1.65 \mathrm{M}_{\odot}$ :

$$
\frac{\mathrm{BE}}{M} \simeq(0.60 \pm 0.05) \beta\left(1-\frac{\beta}{2}\right)^{-1}
$$

Using the radius constraints implied by the experimental limits to $S_{v}$ and $L$ implies BE $\simeq 279_{-25}^{+35}$ B for a 1.4 $\mathrm{M}_{\odot}$ neutron star, where $1 \mathrm{~B}$ is $10^{51}$ ergs. 


\subsection{Disk Masses and Energetics of Compact Star Mergers}

Black hole-neutron star mergers are among the most likely progenitors of short gamma-ray bursts (Lee and Ramirez-Ruiz 2007), sources of gravitational radiation (see, for example, (Abadie et al. 2010, Somiya 2012), and sites for the astrophysical r-process (Lattimer and Schramm|1976; Lattimer et al. 1977). Gamma-ray bursts and r-process production are most likely in the case the neutron star is tidally disrupted, and radioactive decay in unbound neutron-rich ejecta could produce an isotropic optical (Roberts et al.2011, Metzger and Berger 2012) or infrared (Kasen, Badnell and Barnes 2013) transient. The potential for mass ejection depends on whether or not tidal effects are strong enough for the neutron star to be disrupted before reaching the innermost stable circular orbit $\left(R_{I S C O}\right)$ of the black hole. In this case, some disrupted matter remains outside the black hole for times up to seconds in an accretion disk, a tidal tail or in unbound matter. Primarily, the outcome depends on the mass ratio of the binary $\left(q=M_{B H} / M_{N S}\right)$, the spin of the black hole $\left(\chi=a / M_{B H}\right)$, and the neutron star compactness $(\beta)$. Low mass ratios, high spins, and large neutron star radii are favorable for large disk masses. Recently, Foucart (2012) has performed a study of numerical simulations in which these parameters were varied and formulated a two-parameter model that predicts the disk mass remaining outside the black hole a few milliseconds following a merger. The observed energy of a short gamma-ray burst (Giacomazzo et al. 2013) and, possibly, the mass of unbound matter, may be proportional to the disk mass, so observations of gamma-ray bursts can be compared to models of population synthesis of compact binaries in support of this hypothesis.

The fit of Foucart (2012) predicts that the disk mass fraction $M_{t} / M_{N S}$ resulting from a merger varies with $q, \beta$ and $\chi$ according to

$$
\frac{M_{t}}{M_{N S}}=\frac{M_{N S}^{b}}{M_{N S}}\left[a_{1}(3 q)^{1 / 3}(1-2 \beta)-a_{2} \beta q \frac{R_{I S C O} c^{2}}{G M_{B H}}\right],
$$

where $M_{N S}^{b}$ is the baryon mass of the neutron star and $a_{1}=0.288 \pm 0.011$ and $a_{2}=0.148 \pm 0.007$ are parameters determined by a fit to merger simulations. The radius $R_{I S C O}$ of the innermost stable circular orbit depends on $\chi$ according to

$$
\chi=\frac{1}{3} \sqrt{\frac{R_{I S C O c^{2}}}{G M_{B H}}}\left[4-\sqrt{3 \frac{R_{I S C O c^{2}}}{G M_{B H}}-2}\right],
$$

valid for either prograde or retrograde orbits. We also note that Eq. 32 implies that

$$
\frac{M_{N S}^{b}}{M_{N S}} \simeq 1+\frac{3}{5} \frac{\beta}{1-\beta / 2} .
$$

Foucart (2012) argues that the major effects of general relativity are taken into account by the factor $1-2 \beta$ and the dependence of $R_{I S C O}$ on $M_{B H}$ and $\chi$.

Eq. (33) can be expressed in the form $\chi=\chi\left(q, \beta, M_{t}, M_{N S}\right)$. Foucart (2012) presented results as a series of figures illustrating the ratio of disk mass to $M_{N S}^{b}$ as a function of the three variables $q, \beta$ and $\chi$. This fourdimensional function can be reduced to essentially two dimensions by employing experimental restrictions 
to the nuclear symmetry energy which confine neutron star radii to small ranges. Given that the range of $L$ values deduced from experiments is nearly the same as that inferred from astrophysical modeling, we use the baseline results from Steiner, Lattimer and Brown (2010, 2013) that $11<R<12.5 \mathrm{~km}$ is valid not only for $1.4 \mathrm{M}_{\odot}$ neutron stars, but also for stars in the range 1.2-1.8 $M_{\odot}$. We further restrict the likely values of $M_{N S}$ to this range. With these constraints, $0.14 \lesssim \beta \lesssim 0.24$ and Eq. (33) therefore implies a small range of $\chi$ values for particular choices of $q$ and $M_{t}$. As a result, the series of diagrams shown in Foucart (2012) can be distilled into a single picture (Fig. 4). We find the resulting width of $\chi$ bands corresponding to given $M_{t}$ values is minimized if the abscissa is chosen to be $q \beta^{2}$ (justified below). Nevertheless, due to the restricted range of $\beta$, the abscissa is still dominated by its $q$ dependence. Generally, the bottom (top) of each $\chi$ band in Fig. 4 corresponds to $M_{N S}=1.2(1.8) M_{\odot}$ and $R=12.5(11) \mathrm{km}$. The region to the right of the $M_{t}=0$ band corresponds to the situation that tidal disruption occurs within the ISCO so that no disk is able to form and no short gamma-ray burst will be observed. It is readily seen that the disk mass increases rapidly with black hole spin, and that maximally spinning holes of virtually any stellar mass will form disks after merging. The disk mass also increases with decreasing $q$ or $\beta$. This behavior is easily understood if Eq. (33) is rewritten as

$$
r \equiv \frac{R_{I S C O} c^{2}}{G M_{B H}}=\frac{a_{1}\left(q \beta^{2}\right)^{1 / 3} \beta^{1 / 3}(1-2 \beta)-\left(M_{t} / R\right)\left(M_{N S} / M_{N S}^{b}\right)}{a_{2} q \beta^{2}}
$$

We note that $0.32 \lesssim \beta^{1 / 3}(1-2 \beta) \lesssim 0.37$ and $0.86 \lesssim M_{N S} / M_{N S}^{b} \lesssim 0.92$ are weak functions of $\beta$ in the relevant range, so that $r$ is largely a function of $M_{t}$ and $q \beta^{2}$ alone. Also recall that $r$ is a decreasing function of $\chi=a / M_{B H}$. Clearly, $r$ decreases and $a / M_{B H}$ increases with increasing $q^{2} \beta$ for small to moderate values of $M_{t}$. However, for large enough values of $M_{t}$, of order $0.5 \mathrm{M}_{\odot}$ or greater, the numerator of Eq. 36 vanishes for small values of $q^{2} \beta$ so that $\chi \approx 1$ for all values of the abscissa.

A further consequence of Eq. [33] is that the mass of the disk and, presumably, the unbound matter will be sensitive to the neutron star compactness and expected to decrease with increasing $\beta$.

The most energetic observed short gamma-ray bursts imply disk masses of order $0.5 \mathrm{M}_{\odot}$ (Giacomazzo et al.2013), which can only be accommodated for black hole-neutron star systems with $\chi \simeq 1$. Alternatively, Giacomazzo et al. (2013) suggest that binary neutron star mergers could more likely produce large disk masses. They estimated from general relativistic simulations using idealized equations of state that

$$
M_{t} \approx 3(1-q)\left(1+q-M_{t o t} / M_{\max }\right) \simeq 3\left(1-q^{2}\right)\left(1-M_{1} / M_{\max }\right),
$$

where $q<1$ is the binary mass ratio, $M_{t o t}$ is the total gravitational mass of the two neutron stars, and $M_{\text {max }}$ is the maximum mass of an isolated neutron star. This formula predicts that a disk mass of order 0.5 $\mathrm{M}_{\odot}$ could be produced if $q$ is significantly different from 1 and if the larger neutron star is considerably less massive than the maximum mass. However, Eq. (37) is suspect because it is based on simulations employing idealized fluid EOSs, which have unphysical $M-R$ relations; in addition, the dependence on neutron star compactness was not considered. It can be expected, in particular, that the disk mass should decrease with compactness as was already noted for black hole-neutron star mergers above. In fact, this inverse behavior is observed for the mass of unbound matter in the extensive set of general relativistic simulations employing realistic EOSs produced by Bauswein, Goriely and Janka(2013). 


\subsection{The Core-Crust Boundary and the Crust Thicknesses}

In addition to neutron star radii and moments of inertia, several properties of the neutron star crust are also impacted by the nuclear symmetry energy. The most important effect is the location of the core-crust boundary, where a phase transition separates heterogeneous matter containing nuclei and a neutron fluid, and homogeneous, uniform nuclear matter. It has been demonstrated that the thickness of the crust, $\Delta R$, defined as the distance between neutron drip density and the core-crust transition density, depends primarily on the stellar radius and the neutron chemical potential at the core-crust boundary (Lattimer and Prakash 2007):

$$
\Delta R / R \simeq(\mathcal{H}-1)\left[\mathcal{H}(1-2 \beta)^{-1}-1\right]^{-1} .
$$

In this expression, $\mathcal{H}=\exp \left[2\left(\mu_{n t}-\mu_{n 0}\right) / m_{b} c^{2}\right]$, where $\mu_{n t}$ and $\mu_{n 0} \simeq-9 \mathrm{MeV}$ are the neutron chemical potential at the core-crust transition and at the stellar surface, respectively. The crust thickness, together with its transport properties, determines the thermal relaxation time of the crust, which is important in in the cooling of young neutron stars (Yakovlev and Pethick 2004), such as the recently detected rapid cooling of Cassiopeia A (Heinke and Ho 2010; Page et al. 2011) and in the cooling of soft X-ray transients (Page and Reddy 2012).

A related quantity is the fraction of the star's moment of inertia residing in the crust, $\Delta I / I$, which depends primarily on the stellar mass and radius and the pressure at the core-crust boundary, and scales as $p_{t} R^{4} M^{-2}$ (Link, Epstein and Lattimer 1999). The standard model for pulsar glitches holds that they are due to the neutron superfluid in the star's crust. In this case, the observed glitch rates and magnitudes for the Vela pulsar imply that

$$
\Delta I / I \gtrsim 0.016,
$$

and similar or smaller values are found for other glitching pulsars (Andersson et al.|2012).

An approximate method of determining the location of the core-crust boundary is by considering the stability of a homogeneous fluid of nucleons in beta equilibrium. Baym, Bethe and Pethick (1971) showed that small density fluctuations in an otherwise uniform fluid lead to instability when

$$
\mu_{p p}-\mu_{n p}^{2} \mu_{n n}^{-1}+4 e \sqrt{\pi \eta}-4 \eta \alpha\left(9 \pi n_{p}^{2}\right)^{1 / 3}=0,
$$

where $\mu_{i j}=\partial \mu_{i} / \partial n_{j}$ and $\mu_{i}$ and $n_{i}$ are the chemical potential and number density, respectively, of neutrons, protons or electrons. $\alpha \simeq 1 / 137$ is the fine-structure constant. $\beta$ is determined by density gradient terms in the nuclear Hamiltonian and can be approximated by $\eta=D\left[1-4 \mu_{n p} / \mu_{n n}+\left(\mu_{n p} / \mu_{n n}\right)^{2}\right]$ where $D \simeq 100 \mathrm{MeV}$ $\mathrm{fm}^{5}$ (Hebeler et al. 2013). It has been assumed that electrons are relativistic and degenerate. The chemical potentials and derivatives in Eq. (40) are equivalent to

$$
\begin{aligned}
\mu_{n} & =\frac{\partial(n e)}{\partial n}-x \frac{\partial e}{\partial x}, \quad \mu_{p}=\frac{\partial(n e)}{\partial n}+(1-x) \frac{\partial e}{\partial x}, \\
\mu_{n n} \mu_{p p}-\mu_{n p}^{2} & =\frac{1}{n} \frac{\partial^{2}(n e)}{\partial n^{2}} \frac{\partial^{2} e}{\partial x^{2}}-\left(\frac{\partial^{2} e}{\partial n \partial x}\right)^{2}, \\
\mu_{n p} & =\frac{\partial^{2}(e n)}{\partial n^{2}}+(1-2 x) \frac{\partial^{2} e}{\partial n \partial x}-\frac{x(1-x)}{n} \frac{\partial^{2} e}{\partial x^{2}},
\end{aligned}
$$




$$
\mu_{n n}=\frac{\partial^{2}(e n)}{\partial n^{2}}-2 x \frac{\partial^{2} e}{\partial n \partial x}+\frac{x^{2}}{n} \frac{\partial^{2} e}{\partial x^{2}} .
$$

Obviously, the functional form of the energy per baryon $e(n, x)$ will influence the core-crust transition density $u_{t}=n_{t} / n_{s}$ determined by Eq. (40). We assume a quadratic symmetry energy with $S_{2}(u)$ given by Eq. (17), and two different functional forms for the symmetric matter energy,

$$
\begin{aligned}
& e(u, 1 / 2)=\left(3 T_{0} / 5\right) u^{2 / 3}[1+c u]+a_{0} u+b_{0} u^{4 / 3}, \\
& e(u, 1 / 2)=-B+(K / 18)(u-1)^{2}+k(u-1)^{3}+d_{0}(u-1)^{4},
\end{aligned}
$$

where

$$
a_{0}=-\left[4 B+\frac{6 T_{0}}{5}\left(1-\frac{c}{2}\right)\right], \quad b_{0}=\frac{3 T_{0}}{5}(1-2 c)+3 B, \quad d_{0}=B-\frac{K}{18}+k .
$$

In the above, we assume $B=16 \mathrm{MeV}$ is the bulk binding energy at $n_{s}$ and $K=240 \mathrm{MeV}$ is the incompressibility. The coefficient $d_{0}$ ensures the energy per particle vanishes at zero density, $e(0,1 / 2)=0$. With these two forms, and the five associated with Eq. (17), we have 10 different expressions for modeling nuclear matter. Excluding variations utilizing Eq. (17)iii), we found that the energy and pressure predicted for pure neutron matter can be adequately approximated, as shown in Fig. 5, for the density range of interest for determining the core-crust transition densities. In $\beta$-equilibrium, at these densities, pure neutron matter is very similar to neutron star matter since proton fractions are of order $1 \%$.

Choosing values for $S_{v}$ and $L$ within the overlap region in Fig. 2, as previously described, the above energy expressions can be used to find the core-crust transition density, pressure and neutron chemical potential, $u_{t}, p_{t}$, and $\mathcal{H}_{t}$, respectively. For each EOS model, $u_{t}\left(p_{t}\right.$ and $\left.\mathcal{H}_{t}\right)$ decreases (increase) with $L$ or $S_{v}$ with narrow spreads. When all possible variations are considered, the values of these quantities, and in particular $p_{t}$, are poorly constrained. However, limiting $S_{2}$ to the forms (i) and (ii) of Eq. (17), supported by neutron matter studies, results in relatively tight constraints. Monte Carlo sampling to $90 \%$ confidence limits the transition density to $0.49 \lesssim u_{t} \lesssim 0.60$, the pressure to $0.40 \mathrm{MeV} \mathrm{fm}^{-3} \lesssim p_{t} \lesssim 0.60 \mathrm{MeV} \mathrm{fm}^{-3}$ and $\mathcal{H}$ to $1.043 \lesssim \mathcal{H} \lesssim 1.053$. This represents a considerable improvement over the analysis of Lattimer and Prakash (2001) which considered interactions leading to $0.2 \mathrm{MeV} \mathrm{fm}^{-3} \lesssim p_{t} \lesssim 0.65 \mathrm{MeV} \mathrm{fm}^{-3}$.

In comparison, Hebeler et al. (2013), working directly from the properties of pure neutron matter found $0.48 \lesssim u_{t} \lesssim 0.56$ and $0.41 \mathrm{MeV} \mathrm{fm}^{-3} \lesssim p_{t} \lesssim 0.47 \mathrm{MeV} \mathrm{fm}^{-3}$. Both quantities are within the boundaries of our determinations, consistent with the relative $S_{v}$ and $L$ values implied by neutron matter studies relative to experimental constraints (Fig. 2). Hebeler et al. (2013) also found that $u_{t}$ decreased and $p_{t}$ increased linearly with increasing $L$ or $S_{v}$ values.

The crust thickness of a $1.4 \mathrm{M}_{\odot}$ star, using Eq. [38), the experimental constraints to $S_{v}$ and $L$, forms (i) and (ii) of Eq. (17), and Monte Carlo sampling, can consequently be limited to $\Delta R / R=0.082 \pm 0.008$, or $\Delta R=0.96_{-0.13}^{+0.17} \mathrm{~km}$, with $1 \sigma$ errors.

The fraction of the moment of inertia of the neutron star crust depends primarily on the mass and radius of the star as well as $p_{t}$ and, to a lesser extent, $u_{t}$ or $h_{t}$. Lattimer and Prakash (2007) showed that this fraction is well approximated by

$$
\frac{\Delta I}{I} \simeq \frac{8 \pi p_{t} R^{4}}{3 G M^{2}}\left[\frac{M R^{2}}{I}-2 \beta\right] e^{-4.8 \Delta R / R} .
$$


Applying the experimental constraints to $S_{v}$ and $L$, and using Eq. (27) together with Monte Carlo sampling, we find $\Delta I / I=0.037_{-0.007}^{+0.012}$ for a $1.4 \mathrm{M}_{\odot}$ star. For comparison, the approximation suggested by Link, Epstein and Lattimer (1999), in which the exponential factor in Eq. (44) is replaced by

$$
\left[1+\frac{2 p_{t}}{n_{s} m_{b} u_{t}}\left(\frac{R c^{2}}{G M}-2\right)\left(\frac{R c^{2}}{G M}+7\right)\right]^{-1}
$$

and $I$ by Eq. 28 , yields $\left[M R^{2} / I-2 \beta\right]=(7 / 2)[1-7 \beta / 4]$ and $\Delta I / I=0.042_{-0.008}^{+0.016}$ for a $1.4 \mathrm{M}_{\odot}$ star.

The inferred values of $\Delta I / I$ from observations, as discussed above, appears to be consistent with this estimate. However, this explanation has been called into doubt (Andersson et al. 2012; Chamel 2012) due to entrainment of superfluid neutrons in the crust, which raises their effective masses to several times their bare mass. As a consequence, the inferred lower limit to $\Delta I / I$ would have to be larger by a similar factor, so that

$$
\Delta I / I \gtrsim 0.07
$$

would be required to explain glitches. Only very low-mass neutron stars, with $M \lesssim 1.2 \mathrm{M}_{\odot}$, could satisfy this criterion, calling the standard model for glitches into question.

Link (2013) has pointed out, however, that $I$ in Eqs. (39) and (46) does not necessarily represent the total moment of inertia of the star. Although it is usually assumed that the crust couples to the entire core of the star, this need not be the case. Link (2013) argues that the timescale for the outer core to decouple from the crust is of the order of weeks to years, and could correspond to the observed post-glitch relaxation timescale, which may simply represent the dynamical recovery of the outer core. If, for example, the crust couples to a fraction of the core, the inferred value of $\Delta I / I$ is decreased by this fraction, perhaps a factor of 2 or more. If this is the case, observations would regain consistency with theoretical expectations for 1.4 $\mathrm{M}_{\odot}$, and, perhaps, more massive, stars.

\subsection{Composition of the Neutron Star Interior}

The restrictions on the density dependence of the symmetry energy from experiments and neutron matter theory expectations not only establishes important constraints on neutron star radii, but, coupled with the discovery of a nearly $2 \mathrm{M}_{\odot}$ neutron star (Demorest et al. 2010), considerably reduces uncertainties in predictions for the properties of high-density matter. The results of Steiner, Lattimer and Brown (2010, 2013 suggest that even at $6 n_{s}$ the pressure can be estimated to within a $1 \sigma$ error of about $35 \%$. The maximum mass constraint has also been used to limit the maximum strangeness (e.g., hyperon) fraction in a neutron star interior: Weissenborn, Chatterjee and Schaffner-Bielich (2012) estimates this to be less than $20 \%$ even if the maximum mass of a purely hadronic star is about $3.2 \mathrm{M}_{\odot}$, which is the largest mass allowed by causality if a $1.4 \mathrm{M}_{\odot}$ star has $R \leq 12 \mathrm{~km}$ (Lattimer 2012). The strangeness content would be smaller for less stiff hadronic EOSs and smaller maximum masses. This restriction in the strangeness content of neutron stars is consistent with studies using the chiral quark-meson coupling (Katayama, Miyatsu and Saito 2012) and relativistic mean-field (Bednarek et al.|2012) models. 
Furthermore, many studies rule out even the possibility of hybrid stars (those with a mixed phase of quarks and hadrons, e.g., Dexheimer et al. (2013)). The study of Endo (2011) found no stable hybrid stars with maximum masses greater than $1.8 \mathrm{M}_{\odot}$. In general, the phase boundary can be modeled with either a Maxwell or Gibbs construction, but if the surface tension of quark matter is sufficiently large, Gibbs transitions are not allowed. Many studies indicate that if the phase transition is of the Maxwell type, the onset of quarks immediately destabilizes the star so that the existence of a hybrid star is not possible.

However, some studies do permit hybrid stars that can satisfy the minimum maximum mass constraint. Lenzi and Lugones (2012) find stable hybrid configurations using the Nambu-Jona-Lasinio (NJL) model with the relatively stiff (i.e., large values of $L$ ) GM1 and TM1 nucleon forces. However, when hyperons are included, the ranges of parameter space allowing stable hybrid stars is severely restricted, and the maximum mass can barely exceed $2 \mathrm{M}_{\odot}$. However, they did not investigate stability using nucleon forces with values of $L$ in the range constrained by experiments, which lowers the permitted maximum masses. Weissenborn et al. (2011) has shown that the existence of hybrid quark stars with a mixed phase and having a sufficiently large maximum mass is strongly dependent upon the assumed hadronic EOS, and is limited to small, specific ranges of quark matter parameters. They found a marked sensitivity to the stiffness of the equation of state as determined by the radii of moderate $\left(1.4 \mathrm{M}_{\odot}\right)$ stars, so that less stiff equations of state lead to smaller permitted parameter ranges for stable hybrid stars. Nevertheless, Weissenborn et al. (2011) only considered hadronic interactions with relatively large values of $L(>100 \mathrm{MeV}$ ). Alford, Han and Prakash (2013) also explored the effects of phase transitions in hybrid stars, but also included the dependence on $L$ in their study. They demonstrated that $L$ values in the experimentally preferred range severely restricts models capable of satisfying the observed minimum maximum mass constraint. Their models are parameterized by the transition density $n_{\text {crit }}$, the magnitude of the phase transition, $\Delta(n e)=\mu \Delta n$, and the sound speed $c_{s}^{2}$ which is assumed constant for $n>n_{\text {crit }}$. For example, with $\Delta n=0, n_{t}=2 n_{s}$ and $c_{s}^{2}=c^{2}, L \simeq 40 \mathrm{MeV}$ could still result in maximum masses of order $2.2 \mathrm{M}_{\odot}$. However, increasing $\Delta(n e)$ to $0.2(n e)_{\text {crit }}$ and $n_{\text {crit }}$ to $4 n_{s}$ reduces the maximum mass to less than $2 \mathrm{M}_{\odot}$. And if $c_{s}^{2}=1 / 3$, the value expected asymptotically for quark matter, no value of $\Delta(n e)$ can satisfy the maximum mass constraint for $n_{\text {crit }} \gtrsim 1.5 n_{s}$.

\section{Conclusions}

Our results indicate that there is emerging concordance among experimental, theoretical and observational studies for values of the nuclear symmetry energy parameters and neutron star radii. We showed that existing estimates of the symmetry parameters from nuclear experiments measuring neutron skin thicknesses, dipole polarizabilities and giant dipole resonances, and from heavy ion collisions, can be made more precise by including the powerful constraints available from nuclear mass fitting. We obtained joint limits to the symmetry parameters from combining the experimental results: $29.0 \mathrm{MeV}<S_{v}<32.7 \mathrm{MeV}$ and 40.5 $\mathrm{MeV}<L<61.9 \mathrm{MeV}$. From theoretical studies of the correlation between neutron star pressures near the nuclear saturation density and neutron star radii, we determine that $R_{1.4} \simeq 11.9 \pm 1.2 \mathrm{~km}$ to $90 \%$ confidence. Independently, information from theoretical neutron matter studies and observations of neutron star masses and radii restrict the neutron star radius for stars in the vicinity of $1.4 \mathrm{M}_{\odot}$ to a somewhat smaller range, 11 
$\mathrm{km}<R_{1.4}<12.5 \mathrm{~km}$ (see (Steiner, Lattimer and Brown 2010; Hebeler et al. 2010; Gandolfi, Carlson and Reddy 2012)). This reinforces the validity of our experimental constraints for the symmetry energy and suggests that uncertainties in the properties of matter near the nuclear saturation density, and in the estimated neutron star radius, can be further reduced by future experiments and observations.

There have been other recent studies with similar goals. Those pertaining to observations of neutron star masses and radii were summarized in the preceding section. In addition, numerous studies have utilized experimental studies to constrain $S_{v}$ and $L$. Most of these studies focused on the implications of single classes of experiments.

Newton et al. (2011) focused on studies of neutron skin thicknesses, isospin diffusion, isobaric analog states, pygmy dipole resonances, optical model potentials, and droplet model predictions. They also included some aspects of the correlations resulting from nuclear binding energies that we have discussed. However, those correlations did not include error bars in several cases and were based on specific force models rather than the generic correlation we attempted to establish. They also discussed implications for the neutron star radius, moment of inertia, the core-crust transition density and pressure, the inner crust-mantle transition density and the fractional crust thickness and moment of inertia. They found that the crust-core transition density decreases with the assumed value of $L$ and that the transition pressure increases with $L$. The possible ranges of $u_{t}$ and $p_{t}$ they found, however, were $0.50 \lesssim u_{t} \lesssim 0.75$ and $0.35 \mathrm{MeV} \mathrm{fm}^{-3} \lesssim 0.85 \mathrm{MeV}-\mathrm{fm}^{-3}$, respectively, both much wider than the ranges we inferred. They discussed implications for the possible extent of pasta phases, a subject we did not explore. Their results for the fractional crust thickness and moment of inertia also showed broader ranges than ours. Finally, they considered the fundamental and overtone frequencies from the spectrum of torsional shear oscillations in the stellar crust. However, the comparison with data is difficult because of superfluid entrainment effects and assumptions concerning the phase of the mantle, as well as the extent of coupling of crustal oscillations with the core. Due to these extensive uncertainties, we have chosen not to consider these frequencies. Finally, Newton et al. (2011) considered the $L$ dependence of the maximum quadrupole ellipticity of the crust which sets an upper limit to the signal that gravitational wave detectors can observe.

Tsang et al. (2012) also recently undertook a comprehensive analysis of heavy-ion collisions, isobaric analog states, nuclear binding energies, pygmy dipole resonances, neutron skin thicknesses, electric dipole polarizabilities, neutron matter, and microscopic nuclear models in an attempt to constrain the symmetry energy parameters. However, their analysis differs significantly from our study. First, their discussion of nuclear binding energies was limited to the results of the finite range droplet model FRDM12(Möller et al. 2012) and did not include the correlation of symmetry parameters established here and studied in, for example, Kortelainen et al. (2010) or Oyamatsu and lida (2003). The best fit values for $S_{v}$ and $L$ for the UNEDF0, UNEDF1, FRDM90, FRTF or LD models we show in Figure 1 do not lie within the error region they indicated for FRDM12, showing that the size of this error region is substantially underestimated. Second, Tsang et al. (2012) determined a weighted average for $r_{n p}^{208}$, namely $0.19 \pm 0.03 \mathrm{fm}$, which is slightly larger than we assume. However, they used this result to infer a range of values for $L$ rather than incorporating the $S_{v}-L$ correlation that Chen et al. (2010) established (and we confirmed) for the neutron skin thickness to more tightly constrain both symmetry parameters. Third, Tsang et al. (2012) established 
correlations between $S_{v}$ and $L$ from the neutron matter studies of Gandolfi, Carlson and Reddy (2012) and Hebeler et al. (2010) that are virtually identical to ours, but they did not derive corresponding error regions. Fourth, Tsang et al. (2012) utilized a detailed study (Dutra et al.|2012) of 240 Skyrme interactions, limited to those 5 that survived 11 macroscopic and 4 microscopic constraints, to infer a correlation between $S_{v}$ and $L$. In a sense, this correlation is analogous to the binding energy constraint we emphasized here, but does not have the same properties. Finally, Tsang et al. did not use measurements of the giant dipole resonance to provide additional constraints to $S_{v}$ and $L$. Overall, they concluded that experiments constrain the symmetry parameters to a range of values centered on $S_{v} \approx 32.5 \mathrm{MeV}$ and $L \approx 70 \mathrm{MeV}$, but did not quantify those ranges. Both central values are larger than our preferred values. This is partly due to our rejection of isobaric analog state and pygmy dipole resonance estimates, and partly to our utilization of a more comprehensive study of the constraints available from fitting nuclear masses.

In addition to the role played by the nuclear symmetry energy in crustal properties, the pulsar glitch phenomenon, and in neutron star cooling, its influence on neutron star radii has other far-reaching consequences. The neutron star radius will influence the neutrino emission during supernovae and proto-neutron star formation, and therefore has ramifications for the supernova mechanism and r-process production in the aftermath of supernovae. In addition, the tidal quadrupole polarizability of neutron stars depends on

$R^{5}$ which has important ramifications for gravitational wave signals. The radius also strongly affects the quantity of mass left in disks after a merger event involving neutron stars, which will leave signatures in the energetics of short gamma-ray bursts and also electromagnetic signals (afterglows) due to r-process nucleosynthsis in ejected, unbound matter.

This work was initiated by the U.S. DOE-funded Topical Collaboration at Los Alamos, and was partially supported by the U.S. DOE grant DE-AC02-87ER40317.

\section{REFERENCES}

Abadie, J. et al. (LIGO Scientific), Class. Quant. Grav. 27, 173001 (2010).

Abrahamyan, S., et al., Phys. Rev. Lett. 108, 112502 (2012).

Alford, M. G., Han, S. and Prakash, M., arXiv:1302.4732 (2013).

Andersson, N., Glampedakis, K., Ho, W. C. G. and Espinoza, C. M., Phys. Rev. Lett. 109241103 (2012).

Audi, G., Wapstra, A. H. and Thibault, C., Nucl. Phys. A 729, 337 (2003).

Bauswein, A., Goriely, S. and Janka, H.-T., submitted to ApJ; arXiv:1302.6530(2013).

Bauswein, A. and Janka, H.-T., Phys. Rev. Lett. 108, 011101 (2012).

Baym, G., Bethe, H. A. and Pethick, C. J., Nucl. Phys. A 175, 225 (1971).

Bednarek, I., Haensel, P., Zdunik, J. L., Bejger, M. and Mańka, R., A\&A 543, 157 (2012). 
Bertsch, G. F., Dean, D. J. and Nazarewicz, W., SciDAC Rev. 6, 42 (2007).

Brown, B. A., Phys. Rev. Lett. 85, 5296 (2000).

Carbone, A. et al., Phys. Rev. C 81, 041301 (2010).

Centelles, M., Roca-Maza,X., Vinas, X. and Warda, M., Phys. Rev. Lett. 102, 122502 (2009).

Chamel, N., Phys. Rev. C 85, 035801 (2012).

Chen, L.-W., Ko, C. M. and Li, B.-A., Phys. Rev. C 72, 064309 (2005).

Chen,L.-W., Ko, C. M. and Li, B.-A., Phys. Rev. C 76, 054316 (2007).

Chen, L.-W., Ko, C. M., Li, B.-A. and Xu, J., Phys. Rev. C 82, 024321 (2010).

Danielewicz, P., Nucl. Phys. A 727, 233 (2003).

Danielewicz, P. and Lee, J., Int. J. Mod. Phys. E 18, 892 (2009).

Daoutidis, I. and Goriely, S., Phys. Rev. C 84, 027301 (2011).

Das, C. B., Das Gupta, S., Gale, C. and Li, B.-A., Phys. Rev. C 67, 034611 (2003).

Demorest, P. B., Pennucci, R., Ransom, S. M., Roberts, M. S. E. and Hessels, J. W. T., Nature 467, 1081 (2010).

Dexheimer, V., Steinheimer, J., Negreiros, R. and Schramm, S., Phys. Rev. C87, 015804 (2013).

Ducoin, C., Margueron, J., Providência, C., and Vidaña, I., Phys. Rev. C 83, 045810 (2011).

Dutra, M., Lourenço, O., Sá Martins, J. S., Delfino, A., Stone, J. R. and Stevenson, P. D., Phys. Rev. C. 85, 5201 (2012).

Endo, T., Phys. Rev. C 83, 068801 (2011).

Farine, M., Pearson, J. M. and Rouben, B., Nucl. Phys. A 304, 317 (1978).

Flanagan, E. E. and Hinderer, T., Phys. Rev. D 77, 021502 (2008).

Foucart, F., Phys. Rev. D86, 124007 (2012).

Furnstahl, R. J., Nucl. Phys. A 706, 85 (2002)

Gandolfi, S., Carlson, J. and Reddy, S., Phys. Rev. C 85, 032801 (2012).

Gearheart, M., Newton, W. G., Hooker, J. and Li., B.-A., MNRAS 418, 2343 (2011).

Giacomazzo, B., Perna, R., Rezzolla, L., Troia, E. and Lazzati, D., ApJ Lett. 762, L18 (2013). 
Guillot, S., Rutledge, R. E. and Brown, E. F., ApJ 732, 88 (2011).

Guillot, S., Servillat, M., Webb, N. A. and Rutledge, R. E., arXiv1302.0023 (2013).

Hebeler, K., Lattimer, J. M., Pethick, C. J. and Schwenk, A., Phys. Rev. Lett. 105, 161102 (2010)

Hebeler, K., Lattimer, J. M., Pethick, C. J. and Schwenk, A., arXiv:1303.4662 (2013)

Heinke, C. O., Rybicki, G. B., Narayan, R. and Grindlay, J. E., ApJ 644, 1090 (2006)

Heinke, C. O. and Ho, W. C. G., ApJ 719, 167 (2010).

Hinderer, T., Lackey, B. D., Lang, R. N. and Read, J. S., Phys. Rev. D 81, 123016 (2010).

Ho, W. C. G. et al., MNRAS 375, 821 (2007).

Hurley, K. et al., Nature 434, 1098 (2005)

Kasen, D., Badnell, N. R. and Barnes, J., arXiv1303.5788 (2013).

Katayama, T., Miyatsu, T. and Saito, K., ApJS 203, 22 (2012).

Kortelainen, M. et al., Phys. Rev. C 82, 024313 (2010).

Kortelainen, M. et al., Phys. Rev. C 85, 024304 (2012).

Krasznahorkay, A., Paar, N., Vretenar, D. and Harakey, M. M., Phys. Lett. B 720, 428 (2013).

Lackey, B. D., Kyutoku, K., Shibata, M., Brady, P. R. and Friedman, J. L., Phys. Rev. D 85, 044061 (2012).

Lattimer, J. M. and Schramm, D. N., ApJ210, 549 (1976).

Lattimer, J. M., Mackie, F., Ravenhall, D. G. and Schramm, D. N., ApJ213, 225 (1977).

Lattimer, J. M., in The Structure and Evolution of Neutron Stars, ed. D. Pines, R. Tamagaki and S. Tsuruta (Addison-Wesley, New York), p. 50 (1992).

Lattimer, J. M., in Nuclear Equation of State, ed. A. Ansari and L. Satpathy (World Scientific, Singapore), p. 83 (1996).

Lattimer, J. M., Annu. Rev. Nucl. Part. Sci. 62, 459 (2012).

Lattimer, J. M., Pethick, C. J., Prakash, M. and Haensel, P., Phys. Rev. Lett. 66, 2701 (1991).

Lattimer, J. M. and Prakash, M., ApJ 550, 426 (2001).

Lattimer, J. M. and Prakash, M., Phys. Rept. 442, 109 (2007).

Lattimer, J. M. and Schutz, B. F., ApJ 629, 979 (2005). 
Lattimer, J. M. and Steiner, A. W., submitted to ApJ (2013).

Lattimer, J. M. and Swesty, F. D., Nucl. Phys. A 535, 331 (1991).

Lee, W. H. and Ramiriz-Ruiz, E., New J. Phys. 9, 17 (2007).

Leahy, D. A., Morsink, S. M. and Chou, Y., ApJ 742, 17 (2011).

Lenzi, C. H. and Lugones, G., ApJ 759, 57 (2012).

Li, T. et al., Phys. Rev. C 81, 034309 (2010).

Link, B., arXiv1211.2209 (2013).

Link, B., Epstein, R. I. and Lattimer, J. M., Phys. Rev. Lett. 83, 3362 (1999).

Lipparini, E. and Stringari, S., Phys. Rep. 175, 103 (1989).

Lyne, A. G., et al., Science 303, 1153 (2004).

Maselli, A., Cardoso, V., Ferrari, V., Gualtieri, L. and Pani, P., arXiv:1304.2052 (2013).

Metzger, D. and Berger, E., ApJ 746, 48 (2012).

Möller, P., Myers, W. D., Sagawa, H. and Yoshida, S., Phys. Rev. Lett. 108, 052501 (2012).

Myers, W. M. and Swiatecki, W. J., Nucl. Phys. A 81, 1 (1966).

Myers, W. M., and Swiatecki, W. J., Ann. Phys. 55, 395 (1969).

Myers, W. M. and Swiatecki, W. J., Ann. Phys. 204, 401 (1990).

Newton, W. G., Gearheart, M., Hooker, J., and Li, B.-A., in "Neutron Star Crust", ed. C. A. Bertulani and J. Piekarewicz, arXiv:1112.2018(2011).

Nikolov, N., Schunck, N., Nazarewicz, W., Bender, M. and Pei, J., Phys. Rev. C 83, 034305 (2011).

Oyamatsu, K. and Iida, K., Nucl. Phys. A 718, 36 (2003).

Özel, F., Baym, G. and Güver, T., Phys. Rev. D 82, 101301 (2010).

Özel, F., Gould, A. and Güver, T. ApJ 748, 5 (2012).

Page, D., Prakash, M., Lattimer, J. M. and Steiner, A. W., Phys. Rev. Lett. 106, 081101 (2011).

Page, D. and Reddy, S., Thermal and Transport Properties of the Neutron Star Inner Crust, in Neutron Star Crust, ed. C. A. Bertulani and J. Piekarewicz, arXiv:1201.5602.

Prakash, M., Ainsworth, T. L. and Lattimer, J. M., Phys. Rev. Lett. 61, 2518 (1988). 
Piekarewicz, J., Agawal, B. K., Coló, G., Nazarewicz, W., Paar, N., Reinhard, P.-G., Roca-Maza, X. and Vretenar, D., Phys. Rev. C 85, 041302 (2012).

Postnikov, S., Prakash, M. and Lattimer, J. M., Phys. Rev. D 82, 024016 (2010).

Psonis, V. P., Moustakidis, Ch. C. and Massen, S. E., Mod. Phys. Lett. A22, 1233 (2007).

Reinhard, P.-G. and Nazarewicz, W., Phys. Rev. C 81, 051303 (2010).

Roberts, L. F., Kasen, D., Lee, W. H. and Ramirez-Ruiz, E. ApJ 736, 121 (2011).

Roberts, L. F., Shen, G., Cirigliano, V., Pons, J. A., Reddy, S. and Woosley, S. E., Phys. Rev. Lett. 108, 061103 (2012).

Roca-Maza, X.,Centelles, M., Viñas, X. and Warda, M., Phys. Rev. Lett. 106, 252501 (2011).

Samuelsson, L. and Andersson, N., MNRAS 374, 256 (2007).

Shetty, D. V., Yennello, S. J. and Souliotis, G. A., Phys. Rev. C 76, 024606 (2007).

Somiya, K., Class. Quant. Grav. 29, 124007 (2012)

Steiner, A. W., Phys. Rev. C 74, 045808 (2006).

Steiner, A. W. et al., Phys. Rep. 411, 325 (2005);

Steiner, A. W., Lattimer, J. M. and Brown, E. F., ApJ 722, 33 (2010).

Steiner, A. W. and Gandolfi, S., Phys. Rev. Lett. 108, 081102 (2012).

Steiner, A. W., Lattimer, J. M. and Brown, E. F., ApJ 765, 43 (2013).

Steiner, A. W. and Watts, A. L., Phys. Rev. Lett. 103, 181101 (2009).

Strohmayer, T. E. and Bildsten, L, in Compact Stellar X-ray Sources, ed. W. Lewin and M. van der Klis (Cambridge University Press, Cambridge, 2004), p. 113.

Suleimanov, V., Poutanen, J., Revnivtsev, M. and Werner, K., ApJ 742, 122 (2011).

Swesty, F. D., Lattimer, J. M. and Myra, E. S., ApJ 425, 195 (1994).

Tamii, A. et al., Phys. Rev. Lett. 107, 062502 (2011).

Thompson, C. and Duncan, R. C., ApJ 561, 980 (2001).

Trippa, L., Coló, G. and Vigezzi, E., Phys. Rev. C 77, 061304 (2008).

Tsang, M. B. et al., Phys. Rev. Lett. 102, 122701 (2009).

Tsang, M. B. et al., Phys. Rev. C 86, 015803 (2012). 
Typel, S. and Brown, B. A., Phys. Rev. C 64, 027302 (2001).

Van Isacker, P. and Dieperink, A. E. L., Int. J. Mod. Phys. E 15, 1371 (2006).

Vidaña, I. Providência, C., Polls, A. and Rios, A. Phys. Rev. C 80, 045806 (2011).

Walter, F. M. et al., ApJ 724, 669 (2010).

Webb, N. A. and Barret, D., ApJ 671, 727 (2007)

Weissenborn, S., Sagert, I., Pagliara, G., Hempel, M. and Schaffner-Bielich, J., ApJ 740, L14 (2011).

Weissenborn, S., Chatterjee, D. and Schaffner-Bielich, J., Phys. Rev. C 85, 065802 (2012).

Xu, J., Chen, L.-W., Li, B.-A. and Ma, H.-R., ApJ 697, 1549 (2009).

Yakovlev, D. G. and Pethick, C. J., Annu. Rev. Ast. \& Ap. 42, 169 (2004).

Yagi, K. and Yunes, N., arXiv1303.1528 (2013).

Zenhiro, J. et al, Phys. Rev. C 82, 044611 (2010).

Zhang, Y., Danielewicz, P., Famiano, M., Li, Z., Lynch, W.G. and Tsang, M. B., Phys. Lett. B 664, 145 (2008). 


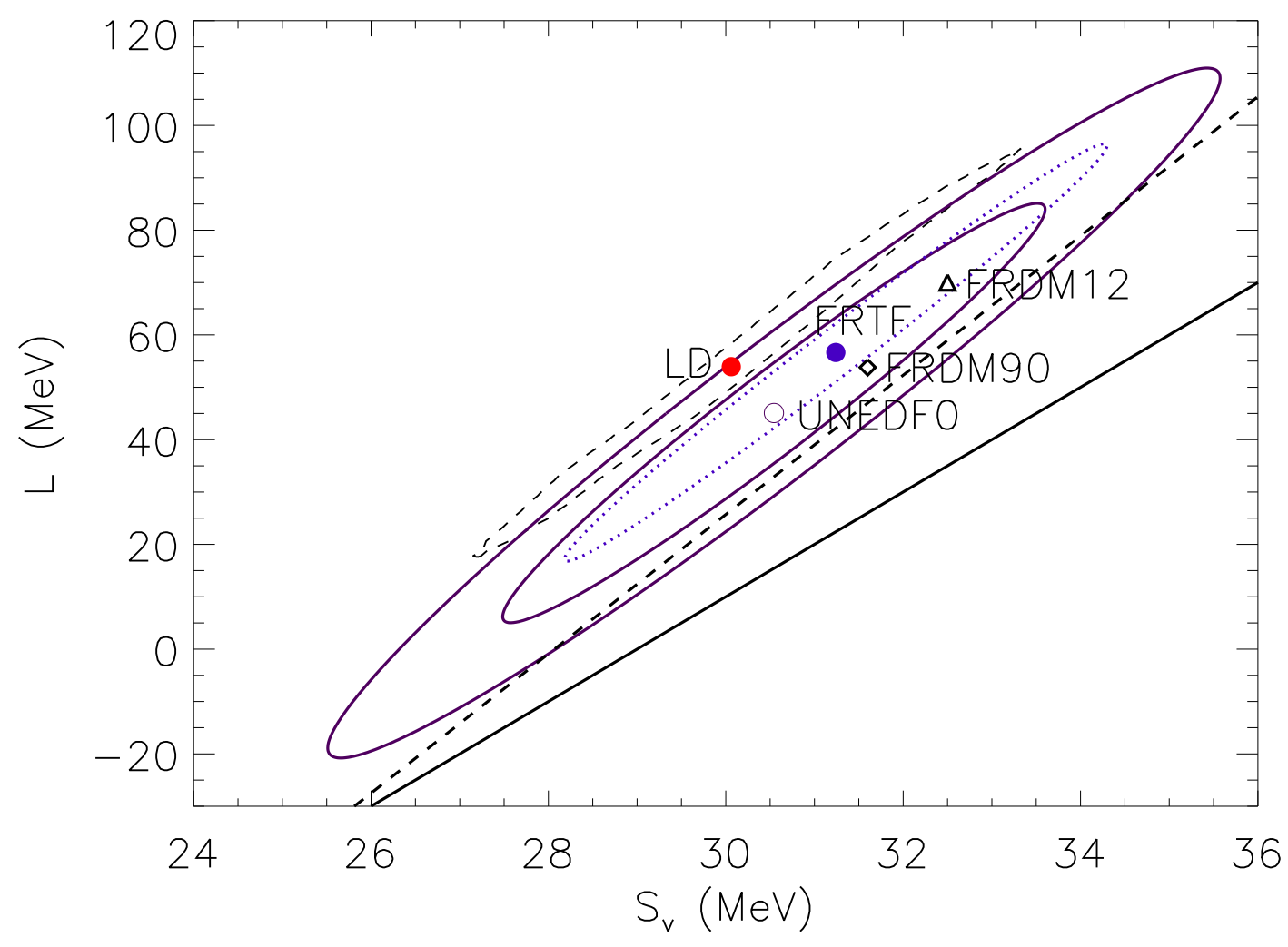

Fig. 1.- Comparison of confidence intervals for nuclear mass fitting. Solid figures are the UNEDF0 68\% and $95 \%$ confidence intervals of Kortelainen et al. (2010) assuming $\sigma_{U N E D F 0}=2 \mathrm{MeV}$. The dashed and dotted figures denote the $68 \%$ confidence intervals for a liquid droplet fit assuming $\sigma_{L D}=0.5 \mathrm{MeV}$ and a Thomas-Fermi finite-range fit assuming $\sigma_{F R T F}=1.6 \mathrm{MeV}$, respectively. Circles mark values of $S_{v}$ and $L$ at the respective $\chi^{2}$ minima. The solid line is the correlation of Farine, Pearson and Rouben (1978) and the dashed line is the correlation of Oyamatsu and Iida (2003). The diamond (Myers and Swiatecki 1990) and triangle (Möller et al.2012) show finite-range liquid droplet mass fits. 


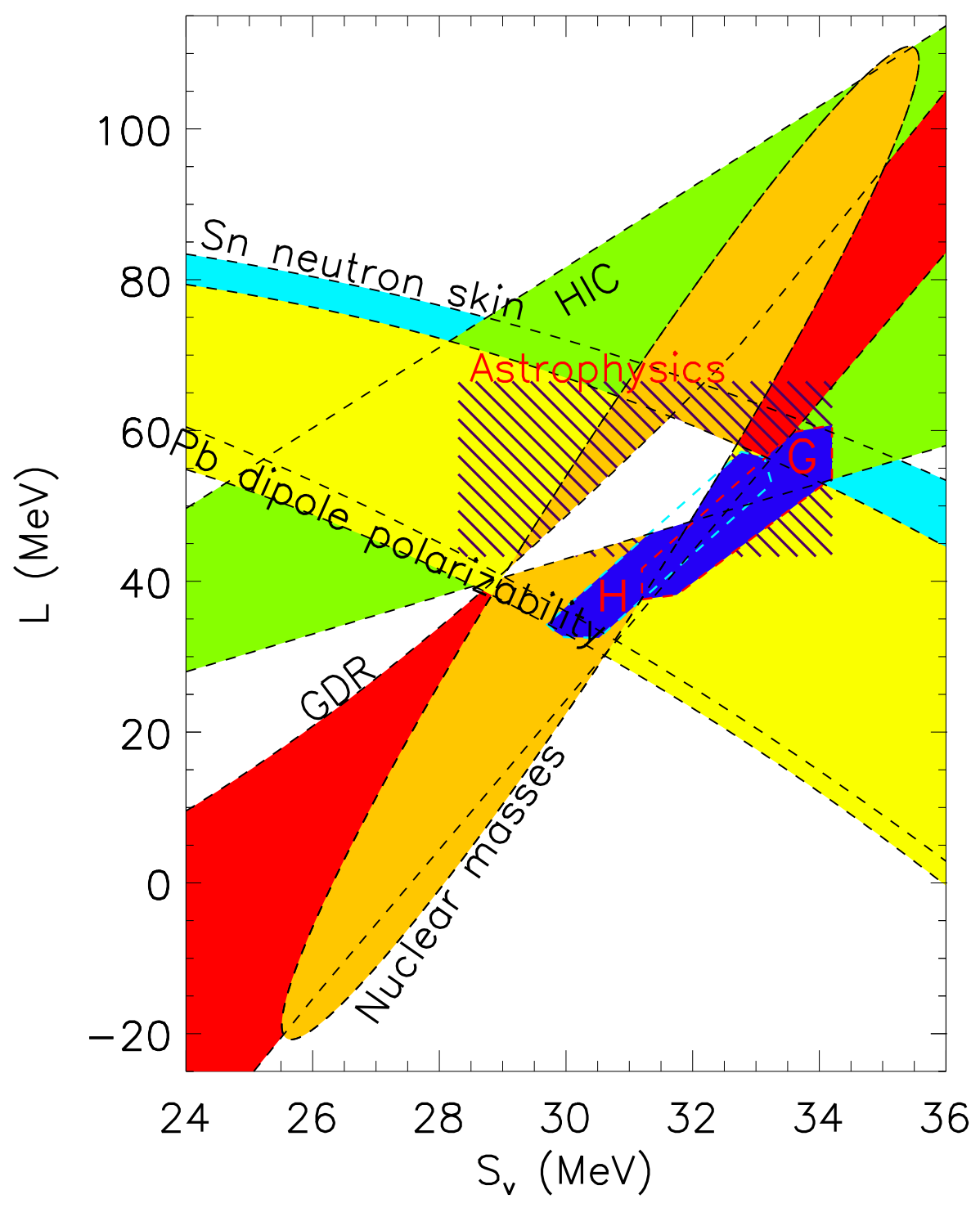

Fig. 2.- Summary of constraints on symmetry energy parameters. The filled ellipsoid indicate joint $S_{v}-L$ constraints from nuclear masses (Kortelainen et al. 2010). Filled bands show constraints from neutron skin thicknesses of Sn isotopes (Chen et al. 2010), the dipole polarizability of ${ }^{208} \mathrm{~Pb}$ (Piekarewicz et al. 2012), giant dipole resonances (GDR) (Trippa, Coló and Vigezzi 2008), and isotope diffusion in heavy ion collisions (HIC) (Tsang et al. 2009). The hatched rectangle shows constraints from fitting astrophysical $M-R$ observations (Steiner, Lattimer and Brown 2010, 2013). The two closed regions show neutron matter constraints ( $\mathrm{H}$ is Hebeler et al. (2010) and $\mathrm{G}$ is Gandolfi, Carlson and Reddy (2012)). The enclosed white area is the experimentally-allowed overlap region. 


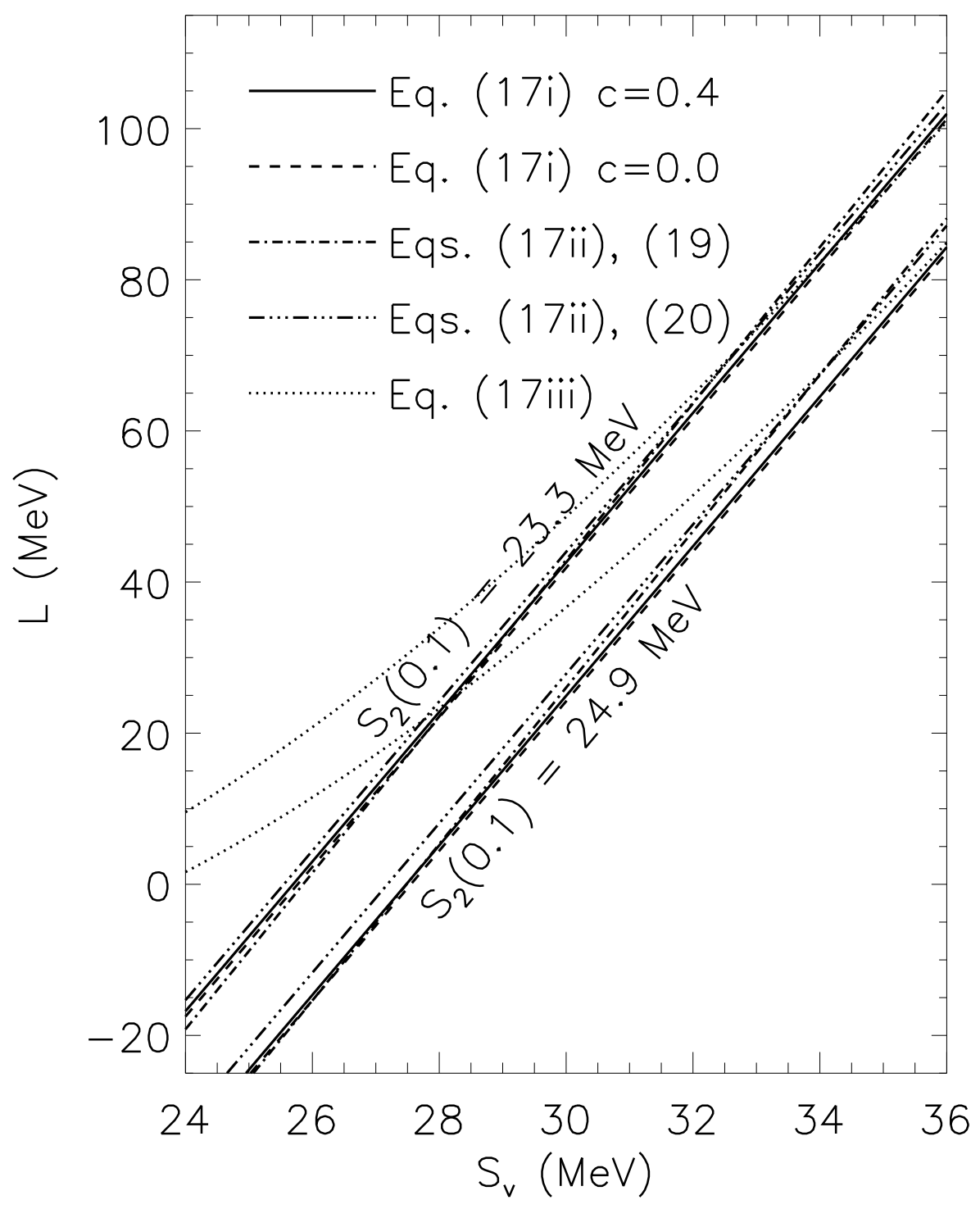

Fig. 3.- Bounds for symmetry energy parameters implied by the constraint $23.3 \mathrm{MeV}<S_{2}(0.1)<24.9$ $\mathrm{MeV}$, derived from giant dipole resonances (Trippa, Coló and Vigezzi 2008), combined with the various forms of Eq. (17) described in the text. 


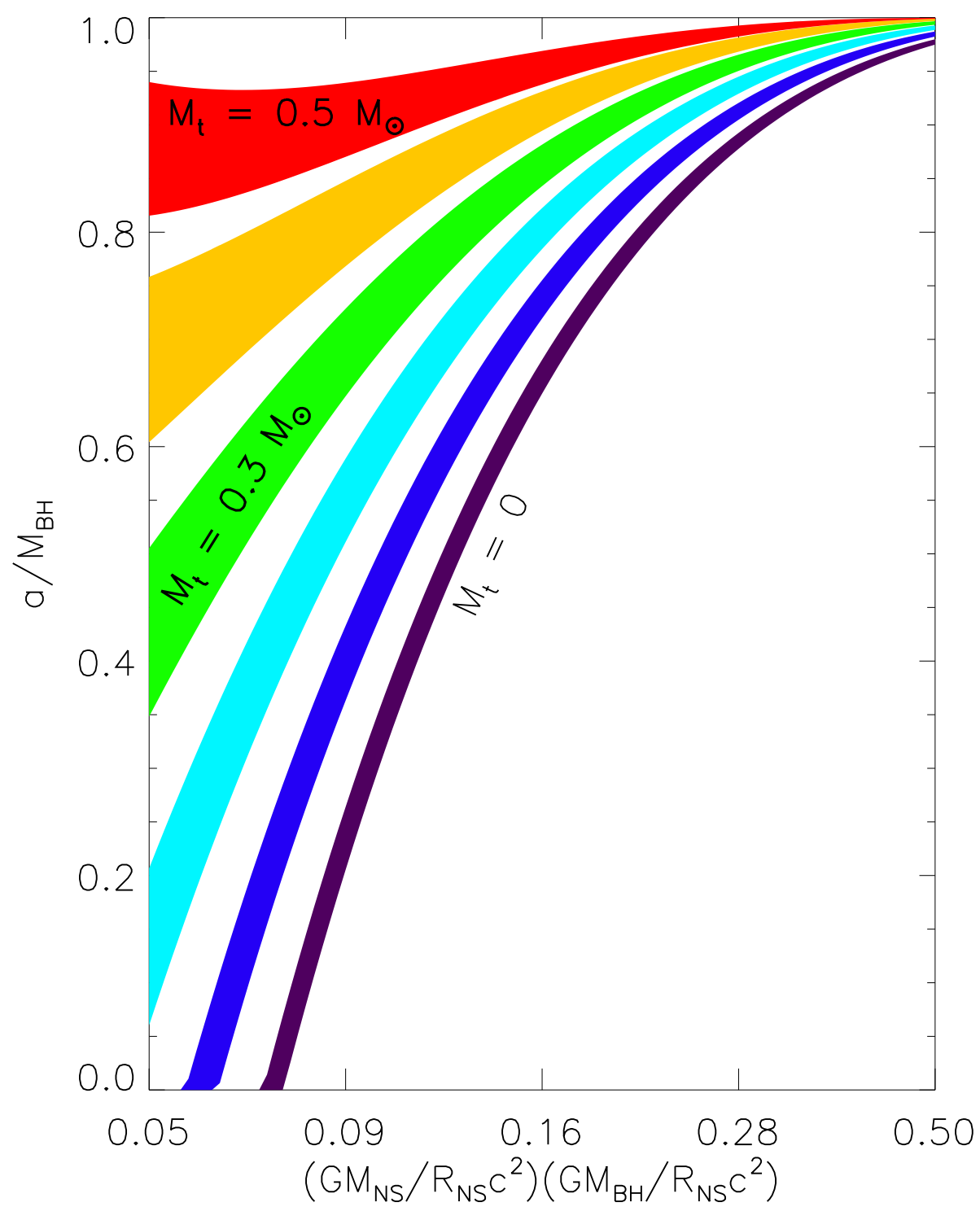

Fig. 4.- Disk mass in the aftermath of a black hole-neutron star merger as predicted by the model of Foucart (2012), from 0 to $0.5 \mathrm{M}_{\odot}$, in increments of $0.1 \mathrm{M}_{\odot}$. The width of the bands represent the effects of varying $R$ and $M_{N S}$ as discussed in the text. Note that the $x$-axis is logarithmic. 


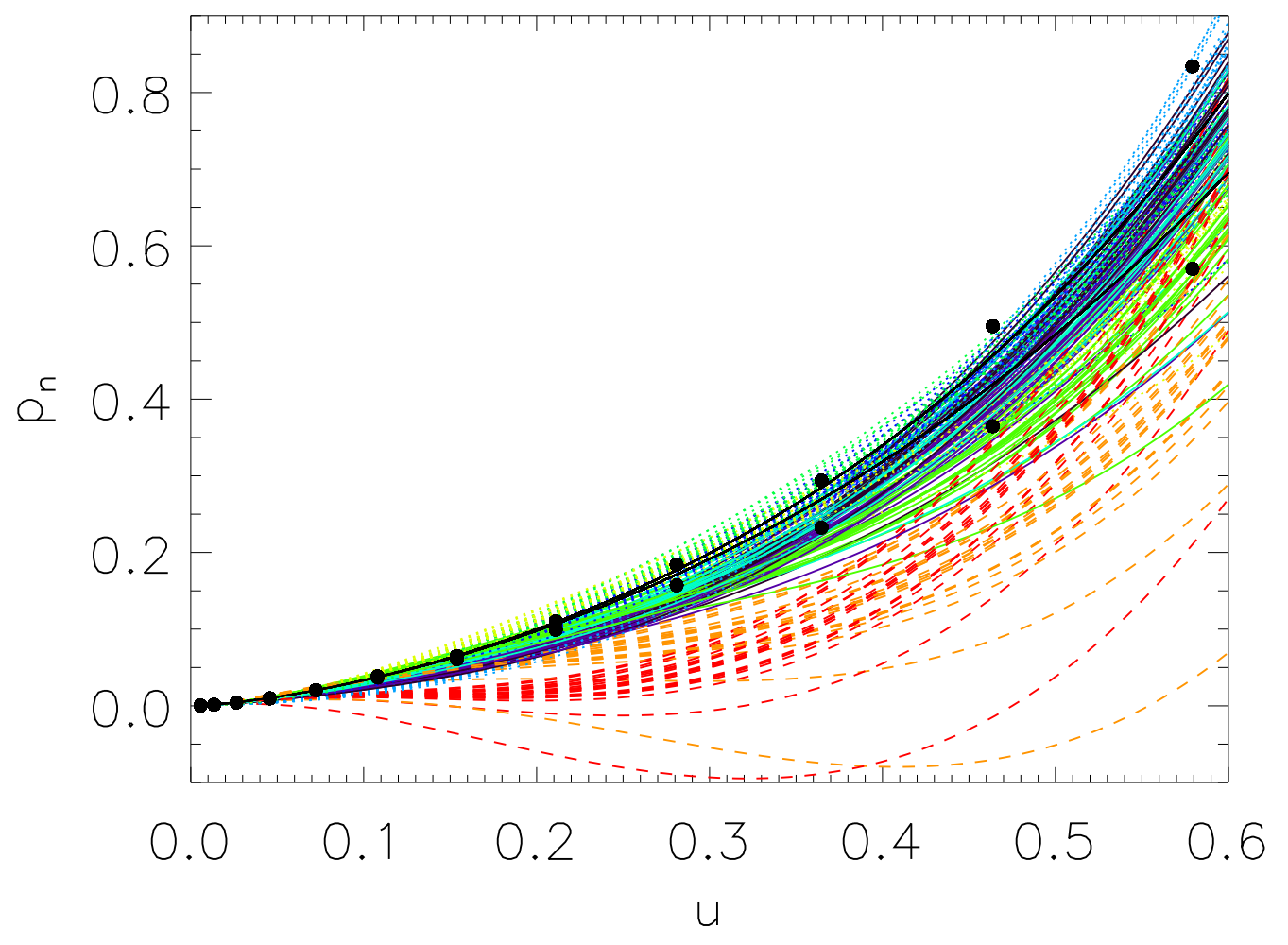

Fig. 5.- Pressures of pure neutron matter as determined from Eq. (42). Solid, dotted and dashed lines correspond to forms i, ii and iii, respectively, for $S_{2}(u)$, randomly choosing force parameters bounded by experimental constraints on $S_{v}$ and $L$ as discussed in the text. Filled circles indicate bounds for neutron matter pressures from (Hebeler et al. 2010). 